\title{
Physical constraints for the evolution of life on exoplanets
}

\author{
Manasvi Lingam* and Abraham Loeb ${ }^{\dagger}$ \\ Institute for Theory and Computation, \\ Harvard University, \\ Cambridge, MA 02138, \\ USA
}

Recently, many Earth-sized planets have been discovered around stars other than the Sun that might possess appropriate conditions for life. The development of theoretical methods for assessing the putative habitability of these worlds is of paramount importance, since it serves the dual purpose of identifying and quantifying what types of biosignatures may exist and determining the selection of optimal target stars and planets for subsequent observations. This Colloquium discusses how a multitude of physical factors act in tandem to regulate the propensity of worlds for hosting detectable biospheres. The focus is primarily on planets around low-mass stars, as they are most readily accessible to searches for biosignatures. This Colloquium outlines how factors such as stellar winds, the availability of ultraviolet and visible light, the surface water and land fractions, stellar flares, and associated phenomena place potential constraints on the evolution of life on these planets.

\section{CONTENTS}

I. Introduction

II. Stellar winds

A. Planetary magnetospheres

B. Atmospheric escape

III. Stellar electromagnetic radiation

A. Evaporation of oceans and buildup of oxygen

B. Origin of life

C. Evolution of complex life

1. Biological damage due to UV radiation

2. Photosynthesis

3. Oxygen and complex life

IV. Stellar flares

A. Electromagnetic radiation

B. Coronal mass ejections

C. Stellar proton events

V. Discussion

Acknowledgments

References

\section{INTRODUCTION}

Furthermore, there are infinite worlds both like and unlike this world of ours. For the atoms being infinite in number ... are borne on far out into space.

- Epicurus (341-270 BC $)^{1}$

\footnotetext{
*manasvi.lingam@cfa.harvard.edu

† aloeb@cfa.harvard.edu

1 The translation of the above quote has been adopted from Bailey (1957).
}

Ever since the ancient Greeks, and probably even earlier, many have argued in favor of the existence of worlds (planets and moons) outside our Solar system. However, it was only a few decades ago that the first extrasolar planets (exoplanets) were detected, owing to the observational challenges associated with finding planets because of their extremely low brightness and masses relative to their host stars. One of the notable early discoveries was 51 Pegasi b, the first exoplanet orbiting a solar-type star (Mayor and Queloz, 1995). Many of the initial discoveries, including 51 Pegasi b, were of exoplanets whose masses were comparable to, or larger than, that of Jupiter. A review of the early developments in exoplanetary science was given by Perryman (2000).

From the 1990s until the launch of NASA's Kepler spacecraft in 2009 (Batalha, 2014; Borucki, 2016), exoplanets were discovered at a steady rate and numbered in the hundreds. In conjunction with the Kepler mission, designed with the express purpose of finding exoplanets, a flotilla of ground-based telescopes have propelled the explosive growth of exoplanetary science (Winn and Fabrycky, 2015). As of April 2019, the number of confirmed exoplanets is over 4000 with planetary systems possessing more than one exoplanet exceeding $650 .^{2}$ Thus, it would be no exaggeration to state that the field of exoplanets represents one of the most rapidly advancing frontiers in astrophysics (Perryman, 2018).

One of the most compelling reasons behind the study of exoplanets entails the prospect of detecting extraterrestrial life beyond Earth. ${ }^{3}$ It is fair to say that the question,

\footnotetext{
2 http://exoplanet.eu/catalog/

3 Exomoons are also of considerable interest as possible abodes for extraterrestrial life, and are likely to be numerous (Heller et al., 2014), but we shall restrict ourselves to exoplanets as they have
} 
"Are we alone?", is one that has resonated with humanity over millenia. The identification of extraterrestrial life is fraught with immense difficulties and challenges, owing to which it is natural to commence searching for "life as we know it" on a planet that shares the basic physical properties of the Earth. Water is conventionally regarded as one of the most essential requirements for life on Earth owing to its unusual physicochemical properties (Ball, 2008). Hence, most studies tend to adopt a "follow the water" approach, i.e. to search for planets where liquid water could exist on their surfaces.

Before proceeding further, we caution that solvents other than water are conceivable and biochemistries not based on carbon are feasible (Benner et al., 2004; Bains, 2004; Schulze-Makuch and Irwin, 2008). One notable example in this category is Titan, which is known to possess methane lakes and seas that contribute to a methanebased hydrological cycle (Hayes, 2016; Mitchell and Lora, 2016). We will not tackle this issue herein, despite its undoubted importance, as our understanding of "life as we do not know it" is much less developed compared to our knowledge of life on Earth. ${ }^{4}$

The "follow the water" approach, from a theoretical and observational standpoint, has led to the identification of the circumstellar habitable zone (HZ), i.e., the annular region around the host star where a planet is theoretically capable of hosting liquid water on its surface. Aside from having a clement surface temperature, the planet needs to retain an atmosphere since water ice transforms directly into gas phase in vacuum. Mars, whose mass is one-tenth that of the Earth, is believed to have lost the majority of its atmosphere in the distant past (Jakosky et al., 2017; Dong et al., 2018a).

It is evident that a smaller and less hotter star would emit less radiation, and therefore its HZ would be situated closer to it. However, the HZ depends not only on stellar properties but also a wide range of planetary properties including, but not restricted to, its atmospheric composition (mixture of greenhouse gases), mass and rotation. The HZ has a long and fascinating history dating back to at least the 19th century, which has been discussed by Gonzalez (2005). The modern formulations of the HZ have matured during the past couple of decades (Kasting et al., 1993; Kopparapu et al., 2013, 2014; Yang et al., 2014; Zsom, 2015; Kopparapu et al., 2016; HaqqMisra et al., 2016; Ramirez, 2018; Schwieterman et al., 2018). Although we focus only on planets within the circumstellar HZ herein, it is worth noting the concept

been subjected to more theoretical and observational studies. Very recently, some preliminary evidence has been presented in favor of a Neptune-sized exomoon candidate orbiting the Jupitersized planet Kepler-1625b (Teachey and Kipping, 2018).

${ }^{4}$ Whenever we shall employ the word "life" henceforth, it must be viewed with the proviso understanding that we actually refer to "life as we know it", unless explicitly stated otherwise. of the $\mathrm{HZ}$ has been extended to encompass both exomoons as well as planets around stellar binaries, i.e., the circumplanetary (Heller et al., 2014; Dobos et al., 2017) and circumbinary (Kane and Hinkel, 2013; Cuntz, 2014) habitable zones, respectively.

There are a couple of important points that must be borne in mind regarding the HZ. First, not all planets within the $\mathrm{HZ}$ are guaranteed to actually have liquid water on the surface. Second, even the presence of liquid water is a necessary, but not sufficient, condition for the existence of life. For example, some of the other necessary requirements include sufficient abundances of "bioessential" elements such as carbon, hydrogen, oxygen, nitrogen and phosphorus as well as free energy flows (Hoehler et al., 2007; Morowitz and Smith, 2007). ${ }^{5}$ Hence, it is essential to avoid conflating the $\mathrm{HZ}$ with the much wider notion of habitability (Moore et al., 2017; Tasker et al., 2017). As per the 2015 NASA Astrobiology Strategy, habitability can be understood in the following terms. ${ }^{6}$

Habitability has been defined as the potential of an environment (past or present) to support life of any kind ... Habitability is a function of a multitude of environmental parameters whose study is biased by the effects that biology has on these parameters.

Further reviews of this multi-faceted subject can be found in Dole (1964); Lammer et al. (2009); Javaux and Dehant (2010); Lineweaver and Chopra (2012); Seager (2013); Cockell et al. (2016); Ehlmann et al. (2016).

Although the above limitations of the HZ definition must be duly recognized, it is equally important to appreciate its strengths. As the HZ depends on a wide range of planetary and stellar parameters, it encompasses a diverse array of potentially habitable environments; an overview of the recent progress in defining and studying HZs was presented by Ramirez (2018). Perhaps, more importantly, owing to the constraints imposed by observation time and funding, the selection of suitable target stars and planets is of paramount importance (Horner and Jones, 2010; Kaltenegger et al., 2010; Lingam and Loeb, 2018a). In this respect, the HZ provides a potential methodology for identifying and selecting planets for more detailed follow-up observations.

In connection with the HZ, a couple of exciting discoveries over the past few years merit a special mention. An exoplanet was discovered in the HZ of Proxima Centauri, the star nearest to the Earth at a distance of $1.3 \mathrm{pc}$, namely, $4 \times 10^{16} \mathrm{~m}$ (Anglada-Escudé et al., 2016). This

\footnotetext{
${ }^{5}$ As life is a far-from-equilibrium phenomenon, it is more accurate to state that the conversion of thermodynamic disequilibria facilitates its emergence (Branscomb et al., 2017).

${ }^{6}$ https://nai.nasa.gov/media/medialibrary/2016/04/NASA_ Astrobiology_Strategy_2015_FINAL_041216.pdf
} 
exoplanet, named Proxima Centauri b (or Proxima $b$ for short), has a minimum mass of $1.3 M_{\oplus}$, where $M_{\oplus}$ is the mass of the Earth. The second major breakthrough entailed the discovery of seven, roughly Earth-sized, planets orbiting the star TRAPPIST- 1 at a distance of $12.1 \mathrm{pc}$ (Gillon et al., 2016, 2017). Of these seven planets, at least three of them reside within the HZ, and are therefore capable of hosting liquid water on the surface. The masses of these seven planets fall within 0.3-1.16 $M_{\oplus}$ and the radii range 0.77-1.15 $R_{\oplus}$ (Delrez et al., 2018; Grimm et al., 2018), where $R_{\oplus}$ is the radius of the Earth. Another discovery worth pointing out is the planet LHS $1140 \mathrm{~b}$, with a radius and mass of $1.4 R_{\oplus}$ and $6.6 M_{\oplus}$, respectively, in the $\mathrm{HZ}$ of a star at a distance of $12 \mathrm{pc}$ (Dittmann et al., 2017).

Before proceeding further, it is essential to articulate the scope and philosophy of this review. It is not feasible to present a comprehensive account of all aspects of habitability due to length constraints. Instead, only a few select topics that have witnessed notable advances within the past decade are tackled. Virtually all factors that we address have a physical basis and pertain to the properties of the host star. This is because we possess a relatively sound understanding of these aspects, and they are arguably less subject to systemic uncertainties.

We will, for instance, not address the issue of whether a given planet can sustain a stable and clement climate over Gyr $\left(10^{9}\right.$ yrs $)$ timescales, despite its relevance for habitability, because the climate depends on a diverse array of factors such as the orbital eccentricity, axial tilt (obliquity), the existence of a large moon (Laskar et al., 1993), surface landmasses and oceans, and biological feedback mechanisms to name a few. Discussions of how some of these variables affect the climate have been given by Kasting (2010); Pierrehumbert (2010); Catling and Kasting (2017a), whereas analyses of the climates of Proxima $\mathrm{b}$ and the TRAPPIST-1 system include Turbet et al. (2016); Wolf (2017); Boutle et al. (2017); Alberti et al. (2017); Turbet et al. (2018); Meadows et al. (2018a); Lincowski et al. (2018). In the same spirit, we will not tackle geophysical and biogeochemical cycles herein owing to their complexity (Sarmiento and Gruber, 2006). Furthermore, the biogeochemistry of planets has not been explored in sufficient detail, despite the fact that it can give rise to feedbacks and buffering over short timescales (Schlesinger and Bernhardt, 2013), which are comparable to those encountered in this review.

Looking beyond planetary and stellar properties, highenergy astrophysical phenomena within the Galaxy also play a vital role in regulating planetary habitability. This has prompted the analysis and identification of the Galactic Habitable Zone (Gonzalez et al., 2001; Lineweaver et al., 2004), but the limits of this region remain subject to uncertainty depending on the constraints adopted (Prantzos, 2008; Gowanlock and Morrison, 2018). Examples of the astrophysical risks for life include high doses of ionizing radiation emanating from quasars, supernovae and gamma ray bursts (Thorsett, 1995; Melott and Thomas, 2011; Piran and Jimenez, 2014; Sloan et al., 2017; Balbi and Tombesi, 2017; Forbes and Loeb, 2018; Lingam et al., 2019).

We mostly focus on planets in the HZ of the most common stars, namely M-dwarfs. Earlier reviews of the habitability of M-dwarf exoplanets can be found in Tarter et al. (2007); Scalo et al. (2007); Shields et al. (2016). Broadly speaking, M-dwarfs are low-mass stars with a mass $M_{\star}$ in the range $0.075<M_{\star} / M_{\odot}<0.5$, where $M_{\odot}$ is the solar mass. M-dwarfs also exhibit significant variation in their radii, effective temperatures, surface magnetic fields and activity (Chabrier and Baraffe, 2000). One of the most notable features of M-dwarfs is that they appear in two distinct "flavors" (Chabrier and Baraffe, 1997; Stassun et al., 2011). M-dwarfs with $M_{\star} \gtrsim 0.35 M_{\odot}$ are characterized by stellar interiors with an inner radiative zone and an outer convective envelope; in contrast, M-dwarfs with $M_{\star} \lesssim 0.35 M_{\odot}$ are fully convective, i.e., they do not possess the radiative zone.

Not only are these low-mass stars the most common and long-lived in the Universe (Tarter et al., 2007; Adams and Laughlin, 1997; Chabrier, 2003), about 20\% of them have Earth-sized planets in their HZs (Bonfils et al., 2013; Dressing and Charbonneau, 2015; Mulders et al., 2015a). These planets are also comparatively accessible to detailed observations (Winn, 2010; Fujii et al., 2018), mostly as a consequence of their smaller orbital radii. Finally, the nearest planets in the HZ described earlier Proxima b, the TRAPPIST-1 system and LHS 1140b are located around M-dwarfs.

The outline of this Colloquium is as follows. In Sec. II, we describe how stellar winds can erode planetary atmospheres and reduce the shielding offered by planetary magnetospheres. The former, in particular, are especially important since they serve as the repositories of most biomarkers, implying that their existence is necessary for detecting non-technological extraterrestrial life outside our Solar system. We discuss how the origin and evolution of life is affected by electromagnetic radiation fluxes from the host star in Sec. III. This is followed by analyzing how stellar flares and their associated physical phenomena influence biospheres in Sec. IV. We conclude with a summary of the central results in Sec. V.

\section{STELLAR WINDS}

Stellar winds are streams of plasma that originates from the outer regions of stellar coronae (Parker, 1958; Priest, 2014). We will discuss two primary aspects by which stellar winds influence planetary habitability. Additional effects include Ohmic heating in the upper atmosphere (Cohen et al., 2014) that may be up to an order of magnitude higher than the heating from extreme ultravi- 
olet (EUV) radiation in the wavelength range of 10-120 nm (Cohen et al., 2018).

\section{A. Planetary magnetospheres}

When exoplanets possess an intrinsic magnetic field, the solar wind plasma will be deflected (Baumjohann and Treumann, 2012). The cavity created by the planetary magnetic field is the magnetosphere. The magnetopause distance $\left(R_{m p}\right)$, which represents the outer boundary of the magnetosphere, serves as a useful proxy for its size. Let us derive its value here.

Suppose that the planet has a pure dipole magnetic field and that its strength is $B_{p}$ at the surface of the planet of radius $R_{p}$. At the magnetopause distance, the corresponding magnetic field $B_{m p}$ is given by

$$
B_{m p}=B_{p}\left(\frac{R_{p}}{R_{m p}}\right)^{3}
$$

provided that only the radial dependence has been retained. The distance $R_{m p}$ is computed by demanding that the magnetic pressure is approximately equal to the solar wind pressure $P_{s w}$. The latter has contributions from the kinetic, magnetic and thermal energies of the solar wind, but it is the first component that typically dominates, i.e. $P_{s w} \approx \rho_{s w} v_{s w}^{2}$, where $\rho_{s w}$ and $v_{s w}$ are the mass density and velocity of the solar wind respectively. Thus, from $B_{m p}^{2} /\left(2 \mu_{0}\right)=P_{s w}$, we obtain

$$
R_{m p}=R_{p}\left(\frac{B_{p}^{2}}{2 \mu_{0} P_{s w}}\right)^{1 / 6}
$$

where $\mu_{0}$ is the vacuum permeability. In actuality, an additional factor of order unity must be introduced for calculating $R_{m p}$ more accurately, due to deviations from an ideal dipole magnetic field (Gombosi, 1998); the characteristic value for the Earth is $R_{m p} \approx 10 R_{\oplus}$ (Kivelson and Russell, 1995). As $P_{s w}$ is subject to temporal variations depending on the planetary orbit and stellar activity, the value of $R_{m p}$ also varies accordingly. For instance, theoretical models indicate that $P_{s w}$ varies by 1-3 orders of magnitude near Proxima $b$, which translates to a variation in $R_{m p}$ by a factor of $2-5$ (Garraffo et al., 2016). The corresponding average magnetopause distance for Proxima b equals $R_{m p} \sim 2-3 R_{\oplus}$, which is smaller than the Earth's magnetosphere by a factor of a few. A similar study has also been undertaken for the planets of the TRAPPIST-1 system (Garraffo et al., 2017) (see also Dong et al. 2018b).

Before discussing why a smaller magnetosphere may prove to be problematic from the standpoint of habitability, it is instructive to understand, via a toy model, why $R_{m p}$ is smaller for lower mass stars. Denoting the stellar mass-loss rate by $\dot{M}_{\star}$ and assuming the resultant wind is spherically symmetric, we have

$$
\dot{M}_{\star}=4 \pi a_{p}^{2} \rho_{s w} v_{s w},
$$

where $a_{p}$ represents the semi-major axis of the planet. Typically, $v_{s w}$ does not vary significantly beyond the immediate vicinity of the star (Fitzpatrick, 2014), implying that $\rho_{s w} \propto \dot{M}_{\star} / a_{p}^{2}$. Let us consider Proxima b once again. The stellar mass-loss rate of Proxima Centauri has been theoretically predicted to be comparable to that of the Sun (Garraffo et al., 2016), and is consistent with observational constraints (Wargelin and Drake, 2002); see, however, Wood et al. (2001). Using this estimate in conjunction with Proxima b's semi-major axis of $0.0485 \mathrm{AU}$, we find that $\rho_{s w}$ should be about 400 times higher near Proxima $b$ than the corresponding solar wind density at the Earth, which is roughly consistent with more detailed numerical calculations (Garraffo et al., 2016; Dong et al., 2017a). A higher value of $\rho_{s w}$ translates to higher dynamic pressure $\left(P_{s w} \propto \rho_{s w}\right)$, thereby leading to a compression of the magnetopause distance.

Furthermore, M-dwarfs are typically characterized by very strong magnetic fields (Reiners and Basri, 2009; Morin et al., 2010; Reiners, 2012) that are 2-3 orders of magnitude higher than the average magnetic field at the Sun's surface $\left(\sim 10^{-4} \mathrm{~T}\right)$. As a result, the stellar wind pressure also includes a significant component from the star's magnetic field (Vidotto et al., 2013). This serves to raise the value of $P_{s w}$, and thereby decrease $R_{m p}$ in accordance with (2). In general, if $P_{s w}$ is 2-3 orders of magnitude higher for planets in the $\mathrm{HZ}$ of low-mass stars, it follows from (2) that the planetary magnetic field $B_{p}$ will need to be higher by a factor of $\mathcal{O}(10)$ in order to yield a magnetopause distance similar to that of the Earth. Hence, for sufficiently high magnetic fields, it is conceivable that planets could possess Earth-sized magnetospheres (See et al., 2014).

However, there are grounds for supposing that $B_{p}$ will be reduced for planets orbiting low-mass stars. As per dynamo theory, the magnetic field strength is expected to scale with the planet's rotation rate $\left(\Omega_{p}\right)$ as

$$
B_{p} \propto \Omega_{p}^{\alpha},
$$

with $\alpha \approx 0.5-1$ for many of the classical dynamo models (Grießmeier et al., 2005; López-Morales et al., 2011). Other dynamo simulations, in contrast, indicate that $B_{p}$ is independent of $\Omega_{p}$ (Christensen, 2010). ${ }^{7}$ The majority, although not necessarily all, ${ }^{8}$ of the planets in the

\footnotetext{
${ }^{7}$ Differentiating between the various dynamo models requires the measurement of exoplanetary magnetic fields. In principle, this could be done through a number of observational avenues based on radio auroral emission, early transit ingress, $\mathrm{H}_{3}^{+}$emission and Ly- $\alpha$ absorption profiles of exoplanets (Grießmeier, 2015).

8 The presence of a thick atmosphere (Leconte et al., 2015), semiliquid interior (Makarov, 2015), or a companion (Vinson and Hansen, 2017) could drive the planet into asynchronous rotation.
} 
HZ of low-mass stars are expected to be synchronous rotators, with their rotation periods equal to their orbital periods, owing to the tidal gravitational force of the host star (Dole, 1964; Bolmont et al., 2011; Barnes, 2017). In this case, the rotation rate will be reduced considerably, and this leads to a corresponding reduction in the magnetic moment (see, however, Zuluaga et al. 2013). For example, a planet $0.2 \mathrm{AU}$ from a star with $M_{\star}=0.5 M_{\odot}$ will have $\Omega_{p} \approx 0.03 \Omega_{\oplus}$, implying that $B_{p} \approx 0.17 B_{\oplus}$ if $\alpha=0.5$ in (4). Here, $\Omega_{\oplus}$ and $B_{\oplus}$ are the Earth's rotation rate and equatorial magnetic field, respectively.

Thus, planets around low-mass stars will typically have smaller magnetospheres on account of increased stellar wind pressure and possibly weaker magnetic moments. Conventionally, weaker planetary magnetic fields and smaller magnetospheres lead to a lower degree of protection against the stellar wind, thereby resulting in enhanced atmospheric escape; we will return to this topic in Sec. II.B. However, this paradigm has been challenged by recent theoretical studies, which indicate that the atmospheric escape rate does not always decline with an increase in the magnetic field strength (Dong et al., 2018c; Blackman and Tarduno, 2018; Gunell et al., 2018; Lingam, 2019). The basic reason can be understood qualitatively as follows. The polar caps are regions close to the magnetic poles with open magnetic field lines, which permit the escape of ions through the polar wind. The latter is dependent on the ambipolar electric field that stems from the difference in the velocities of ions and electrons (Axford, 1968); electrons typically move faster, thus causing charge separation and inducing an electric field, which accelerates the ions and permits their escape (Schunk and Nagy, 2009). The polar wind may become increasingly important for weak dipole magnetic fields with $B_{p} \sim 10^{-7} \mathrm{~T}$ for Mars-like exoplanets (Sakai et al., 2018); see also Lingam (2019).

The second aspect that we highlight concerns the effect of planetary magnetic fields in regulating the amount of cosmic rays that reach the surface. This is particularly relevant since high-energy radiation can drive the radiolysis (decomposition via radiation) of complex biomolecules (Dartnell, 2011; Horvath and Galante, 2012). It is wellknown that the planet's magnetospheric shielding (for deflecting charged particles) influences the amount of radiation that reaches the surface (Grießmeier et al., 2009), but more recent studies have revealed that the dependence on the magnetic moment $\mathcal{M}_{p}$ is very sensitive to the atmospheric column density (Atri et al., 2013). For example, when one considers planets with an atmospheric column density equal to that of the Earth, changing the magnetic moment from $\mathcal{M}_{p}=0$ to $\mathcal{M}_{p}=10 \mathcal{M}_{\oplus}$ (where $\mathcal{M}_{\oplus}$ is the magnetic moment of the Earth) results in the radiation dose rate dropping by a factor of about 6 . In contrast, if the atmospheric column density is about $10 \%$ that of the Earth, the radiation dose rate declines by a factor of 240 as one moves from $\mathcal{M}_{p}=0$ to $\mathcal{M}_{p}=10 \mathcal{M}_{\oplus}$
(Grießmeier et al., 2016).

One other aspect that is not explored here concerns the effect of magnetospheric (and atmospheric) shields on the chemistry and abundances of biosignatures (Grenfell, 2017). Cosmic rays may react with $\mathrm{N}_{2}-\mathrm{O}_{2}$ atmospheres and stimulate the formation of nitrogen oxides $\left(\mathrm{NO}_{x}\right)$ and drive the depletion of ozone $\left(\mathrm{O}_{3}\right)$, the latter of which constitutes a widely studied biosignature. It turns out that high-energy particles produced during stellar flares also have similar effects, as discussed in Sec. IV.C.

\section{B. Atmospheric escape}

Atmospheric escape refers to any mechanism that imparts sufficient energy to particles, enabling them to achieve speeds higher than the threshold to escape the gravitational pull of the planet (Tian, 2015a). Most studies tend to focus on neutral particles, but it is important to appreciate that charged particles can also escape the atmosphere. There are a wide array of mechanisms that enable the escape of ions, and reviews of this subject were given by Lammer et al. (2008) and Brain et al. (2016).

Charged particles are typically accelerated by electric fields in addition to deriving energy from collisions. The electric field $\mathbf{E}$ can be expressed as

$$
\mathbf{E}=-\mathbf{v} \times \mathbf{B}+\frac{\mathbf{J} \times \mathbf{B}-\nabla p_{e}}{n_{e} e}+\eta \mathbf{J}+\ldots,
$$

where $\mathbf{v}$ is the plasma bulk velocity, $\mathbf{J}$ is the current, $\mathbf{B}$ represents the magnetic field, $\eta$ denotes the resistivity, $n_{e}$ is the electron number density and $p_{e}$ is the electron pressure. The above expression is known as Ohm's Law, and contains additional terms that have been omitted here (Freidberg, 2014; Lingam et al., 2016, 2017). Each of the first three terms on the right-hand-side of (5) are known to be capable of accelerating charged particles and enabling them to exit the atmosphere.

Apart from the polar wind encountered in Sec. II.A, which relies on ion outflow from polar regions, there are several other ion escape mechanisms. Ion pick up processes enable the acceleration of ions via electric fields embedded within the stellar wind plasma. In addition, there are a multitude of alternative mechanisms involving plasma instabilities and cool ion outflows (Lammer, 2013). Although, in principle, it is feasible to develop simple models for each of the processes and thus estimate the total loss rate, this approach is not practical within the scope of this review.

Instead, it is instructive to consider a toy model for weakly magnetized or unmagnetized planets, i.e. those that have a negligible intrinsic magnetic field. In our Solar system, Venus falls under this category and so does Mars, although the situation with respect to the latter is more complicated owing to the presence of remnant 
crustal fields (Acuna et al., 1998). As we have seen earlier, weak magnetic fields may be common for planets in the HZ of low-mass stars. Non-thermal escape mechanisms involving ion escape due to interactions with stellar winds could become particularly important for unmagnetized planets that are comparable to the size of the Earth (Dong et al., 2017a; Brain et al., 2016).

Our approach partly mirrors the derivation in Zendejas et al. (2010) and Zahnle and Catling (2017); see also Lingam and Loeb (2018b). To begin with, note that the momentum carried by a single proton in the solar wind is $m_{p} v_{s w}$. In contrast, a chemical species $X$ in the atmosphere requires a maximal momentum gain of $m_{X} v_{e s c}$, where $m_{X}$ is the mass of the particle and

$v_{e s c}=\sqrt{2 G M_{p} / R_{p}}=11 \mathrm{~km} / \mathrm{s}\left(M_{p} / M_{\oplus}\right)^{1 / 2}\left(R_{p} / R_{\oplus}\right)^{-1 / 2}$

is the gravitational escape velocity from the planet. Suppose, for instance, that we consider the loss of $\mathrm{O}_{2}^{+}$as this represents one of the major species lost from $\mathrm{CO}_{2}$ dominated atmospheres; it can be readily verified that $m_{X} \approx 32 m_{p}$. The escape velocity for Earth-sized planets is $\mathcal{O}(10) \mathrm{km} / \mathrm{s}$, whereas $v_{s w}$ is typically $\mathcal{O}(100) \mathrm{km} / \mathrm{s}$, although $\sim 10^{3} \mathrm{~km} / \mathrm{s}$ is also possible. Thus, in heuristic terms, the momentum of a proton in the stellar wind can be wholly imparted to a single entity of $X$ to facilitate the escape of the latter.

Hence, if we determine the rate of protons impinging on the planet, we can also treat this as the escape rate of particles from the planet's atmosphere $\left(\dot{M}_{p}\right)$. Recall that the stellar mass-loss rate is $\dot{M}_{\star}$ and the flux at a distance $a_{p}$ is $\dot{M}_{\star} /\left(4 \pi a_{p}^{2}\right)$. The cross-sectional area presented by the planet is $\pi R_{p}^{2}$. Thus, the escape rate is

$$
\dot{M}_{p}=\frac{1}{4}\left(\frac{R_{p}}{a_{p}}\right)^{2} \dot{M}_{\star} .
$$

In actuality, not all stellar wind protons will contribute to atmospheric escape, owing to which the factor of $1 / 4$ could be replaced with an efficiency factor $\varepsilon$ that can be as much as one order of magnitude lower. If we choose $R_{p}=0.53 R_{\oplus}$ and $a_{p}=1.524 \mathrm{AU}$ for Mars, along with $\dot{M}_{\odot} \sim 2 \times 10^{-14} M_{\odot} \mathrm{yr}^{-1}$ (Wargelin and Drake, 2002), we obtain $\dot{M}_{p} \sim 0.07 \mathrm{~kg} / \mathrm{s}$, which is only about a factor of two lower than the predicted $\mathrm{O}_{2}^{+}$loss rate of $\sim 2.6 \times 10^{24} \mathrm{~s}^{-1}$ (Dong et al., 2018a) that corresponds to $\sim 0.14 \mathrm{~kg} / \mathrm{s}$ (using $\left.m_{\mathrm{O}_{2}^{+}} \approx 32 m_{p}\right) .{ }^{9}$ Numerical simulations carried out by Dong et al. (2018b) indicate that (6) accurately reflects the trend for the expected escape rates of the seven TRAPPIST-1 planets.

Two points regarding (6) are worth mentioning. First, the quantity $\dot{M}_{\star}$ evolves over time, and is much higher

9 At this rate, losing the mass of Earth's atmosphere $\left(\sim 5 \times 10^{18}\right.$ $\mathrm{kg}$ ) would require $\sim 10^{12}$ yrs. when the star is younger (Wood et al., 2005), implying that the atmospheric escape rates will also be correspondingly higher. Second, it is difficult to estimate $\dot{M}_{\star}$ as it depends on a variety of stellar parameters such as the mass, radius and rotation rate (Cranmer and Saar, 2011). A simple theoretical prescription that may be reasonably accurate for stars within the range $0.4<M_{\star} / M_{\odot}<1.1$ is (Johnstone et al., 2015):

$$
\frac{\dot{M}_{\star}}{\dot{M}_{\odot}}=\left(\frac{R_{\star}}{R_{\odot}}\right)^{2}\left(\frac{\Omega_{\star}}{\Omega_{\odot}}\right)^{1.33}\left(\frac{M_{\star}}{M_{\odot}}\right)^{-3.36}
$$

where $M_{\star}, R_{\star}$ and $\Omega_{\star}$ are the stellar mass, radius and rotation rate respectively; it must be noted that (7) applies only to stars with rotation rate $\Omega_{\star}<\Omega_{c}$, where $\Omega_{c}=15 \Omega_{\odot}\left(M_{\star} / M_{\odot}\right)^{2.3}$ with $\Omega_{\odot}$ denoting the solar rotation rate (Johnstone et al., 2015). We note that the validity of (7) is questionable for most M-dwarfs including Proxima Centauri and TRAPPIST-1.

Next, we may rewrite the mass of the atmosphere $\left(M_{a t m}\right)$ in terms of the surface pressure $\left(P_{s}\right)$ via

$$
M_{a t m}=\frac{4 \pi R_{p}^{2} P_{s}}{g}
$$

where $g \approx g_{\oplus}\left(R_{p} / R_{\oplus}\right)^{1.7}$ is the planet's surface gravity (Valencia et al., 2006; Zeng et al., 2016), provided that the planet has a rocky composition and is larger than the size of the Earth. Here, $g_{\oplus}$ denotes the Earth's surface gravity. With this data, we are free to compute the characteristic timescale $\left(t_{p}\right)$ for the depletion of the planet's atmosphere, which is given by $t_{p} \sim M_{a t m} / \dot{M}_{p}$. The advantage is that $t_{p}$ is determined purely in terms of basic physical parameters based on (6), (7) and (8). It has been implicitly supposed that the rate of atmospheric escape is much higher than the rate of outgassing from the mantle due to geological activity.

If we estimate $t_{p}$ for Proxima $\mathrm{b}$ using the above formulae in conjunction with $\dot{M}_{\star} \sim \dot{M}_{\odot}$ and the fiducial (but arbitrary) choice of $P_{s}=1 \mathrm{~atm}$, we find that the timescale is $\mathcal{O}\left(10^{8}\right)$ yrs. This result is in agreement with detailed magnetohydrodynamic numerical simulations of both magnetized and unmagnetized planets that have yielded values ranging between $\mathcal{O}\left(10^{7}\right)$ to $\mathcal{O}\left(10^{9}\right)$ yrs, with many converging on $\mathcal{O}\left(10^{8}\right)$ yrs (Dong et al., 2017a; Airapetian et al., 2017a; Garcia-Sage et al., 2017). In contrast, for the TRAPPIST-1 system, it has been found that the innermost planet (with $P_{s}=1 \mathrm{~atm}$ assumed throughout) may lose its atmosphere over a timescale of $\mathcal{O}\left(10^{8}\right)$ yrs, while the duration of atmospheric retention for the outermost planet is $\mathcal{O}\left(10^{10}\right)$ yrs (Dong et al., 2018b). The timescales for the outer TRAPPIST1 planets are higher than Proxima $b$ because the star TRAPPIST-1 is smaller, less active, and therefore anticipated to have less intense stellar winds.

Other notable studies of atmospheric escape driven by the stellar wind include Kislyakova et al. (2014) and Co- 
hen et al. (2015). An important point worth appreciating here is that planets in the HZ of solar-type (G-type) stars are not likely to lose the entirety of their atmospheres over Gyr or sub-Gyr timescales through ion escape processes (Seki et al., 2001; Dong et al., 2017b). We caution the reader that the above timescales only apply to planets with non-massive atmospheres. If the planets have thick atmospheres - as is possible for the TRAPPIST-1 system (Grimm et al., 2018) - the corresponding timescales would be increased due to $t_{p} \sim M_{a t m} / \dot{M}_{p}$.

Hitherto, we have concerned ourselves with discussing unmagnetized planets when deriving (6). When it comes to magnetized planets, finding an equivalent expression is harder and subject to more uncertainty. It was proposed by Blackman and Tarduno (2018) that the analog of (6) for magnetized planets is given by $\dot{M}_{p}^{(m a g)}=\mathcal{Q} \dot{M}_{p}$, where the extra factor $\mathcal{Q}$ is defined as

$$
\mathcal{Q} \sim 7.1\left(\frac{\chi}{0.1}\right)\left(\frac{R_{m p} / R_{p}}{10}\right)^{2}
$$

where $\chi$ is a parameter that is proportional to the ratio of the speed associated with magnetic reconnection to the stellar wind speed near the planet. However, it should be cautioned that this expression has not yet been validated by numerical simulations. Thus, setting $\chi \sim 0.1$, we find $\mathcal{Q}>1$ is achieved when $R_{m p} \gtrsim 3.75 R_{p}$. Therefore, for magnetized planets that satisfy this criterion, their escape rates may exceed those of their unmagnetized counterparts.

The general conclusion that can be drawn (from the above examples) is that planets in the HZ of very lowmass stars are often, but not always, susceptible to losing their atmospheres over sub-Gyr timescales, even reaching a minimum of $\mathcal{O}\left(10^{7}\right)$ yrs. On Earth, we know that the timescale taken for the origin of life (abiogenesis) was $\leq 0.8$ Gyr (Pearce et al., 2018), and it required 4.5 Gyr for the emergence of technological intelligence, namely, Homo sapiens. Strictly speaking, we have no knowledge whatsoever of what the timescale for abiogenesis is on other planets based on just a single data point from Earth (Spiegel and Turner, 2012). Despite this important caveat, many studies assume that the corresponding timescales are similar on other worlds.

If we operate under this assumption, it seems plausible that Earth-sized planets in the HZ of M-dwarfs with surface pressures comparable to the Earth might not have enough time for biological evolution to take place. Of course, on account of the near-linear dependence of the depletion timescale on $P_{s}$ (Dong et al., 2017a), planets with much thicker atmospheres than our own will retain them over commensurately longer periods. In the event that $t_{p}$ is much less than $t_{H Z}$, i.e. the temporal duration of the $\mathrm{HZ}$, it is the former that will serve as an upper limit on the time over which biological evolution can occur; the expression for $t_{H Z}$ as a function of $M_{\star}$ is found in Rushby et al. (2013) and Lingam and Loeb (2019a). This is because of the fact that the presence of an atmosphere is necessary for sustaining liquid water on the surface as seen from its phase diagram. ${ }^{10}$

Let us suppose that $t_{p}$ is $\mathcal{O}\left(10^{8}\right)$ yrs and that $t_{p} \ll$ $t_{H Z}$. This gives rise to another potential issue. Since the timescale for biological evolution is lower, the biodiversity may also be reduced accordingly. There are some grounds for supposing that the species richness (total number of species) on Earth has grown exponentially over time (Russell, 1983). ${ }^{11}$ Using this approach, Lingam and Loeb (2017a) proposed that planets in the HZ of Mdwarfs could attain a peak species richness that is many orders of magnitude lower than the current-day value of $\sim 10^{12}$ on Earth (Locey and Lennon, 2016), and that M-dwarfs with $M_{\star} \lesssim 0.2 M_{\odot}$ were especially unlikely to host diverse biospheres. Last, we observe that atmospheric erosion will eventually result in lowered column densities, which is problematic because more cosmic rays would reach the surface resulting in enhanced harmful radiation levels, as noted in Sec. II.A.

\section{STELLAR ELECTROMAGNETIC RADIATION}

Of the myriad stellar factors regulating habitability, perhaps the most appreciated amongst them has been the role of electromagnetic radiation emitted by the star. As this represents a vast topic, we will concern ourselves with only a handful of recent developments. An overview of the positive and negative effects on habitability because of electromagnetic radiation has been provided in Table I. We have also depicted the two chief UV fluxes of interest in this paper received by Earth-analogs around low-mass stars as a function of $M_{\star}$ in Figure 1. The phrase "Earth-analogs" should be used with due caution. We refer here to rocky planets that have the same basic physical parameters as the Earth such as the effective temperature, albedo, atmospheric pressure and radius.

\section{A. Evaporation of oceans and buildup of oxygen}

It has been known for a long time that EUV photons are capable of facilitating atmospheric escape (Lammer, 2013). Let us denote the energy flux of EUV photons near the planet by $\mathcal{F}_{E U V}$ and assume that the area over which these photons are intercepted is roughly $\pi R_{p}^{2}$. If these photons enable the particles to attain an escape

${ }^{10}$ In the absence of an atmosphere, surficial life is probably ruled out for the most part, but the existence of subsurface biospheres, which are not tackled herein, is still possible.

11 A more realistic model for the species richness is based on a series of logistic curves spliced together (Purvis and Hector, 2000). 
TABLE I Potential positive and negative biological ramifications arising from electromagnetic radiation

\begin{tabular}{|c|c|c|}
\hline Radiation & Consequences & M-dwarf exoplanets \\
\hline \hline XUV & Water worlds to land-water planets via $\mathrm{H}_{2} \mathrm{O}$ photolysis & High \\
\hline $\mathrm{XUV}$ & Build-up of atmospheric $\mathrm{O}_{2}$ via $\mathrm{H}_{2} \mathrm{O}$ photolysis for complex life & High \\
\hline $\mathrm{UV}-\mathrm{C}$ & Formation of biomolecular building blocks for prebiotic chemistry & Low \\
\hline UV-Bio & Selection agent for evolutionary innovations and speciation & Low \\
\hline PAR & Enabling photosynthesis & Low \\
\hline \hline XUV & Complete desiccation of land-water planets via $\mathrm{H}_{2} \mathrm{O}$ photolysis & High \\
\hline UV-Bio & DNA damage & Low \\
\hline UV-Bio & Inhibiting photosynthesis & Low \\
\hline
\end{tabular}

Notes: XUV $(\sim 0.6-120 \mathrm{~nm})$, UV-C $(\sim 200-280 \mathrm{~nm})$, UV-Bio $(\sim 200-400 \mathrm{~nm})$, PAR $(\sim 400-750 \mathrm{~nm})$.

"High" and "Low" refer to the energy fluxes (in the corresponding wavelength range) received by

Earth-analogs around M-dwarfs relative to that of the Earth. The first five rows correspond to the positives, whereas the last three rows represent the negatives.

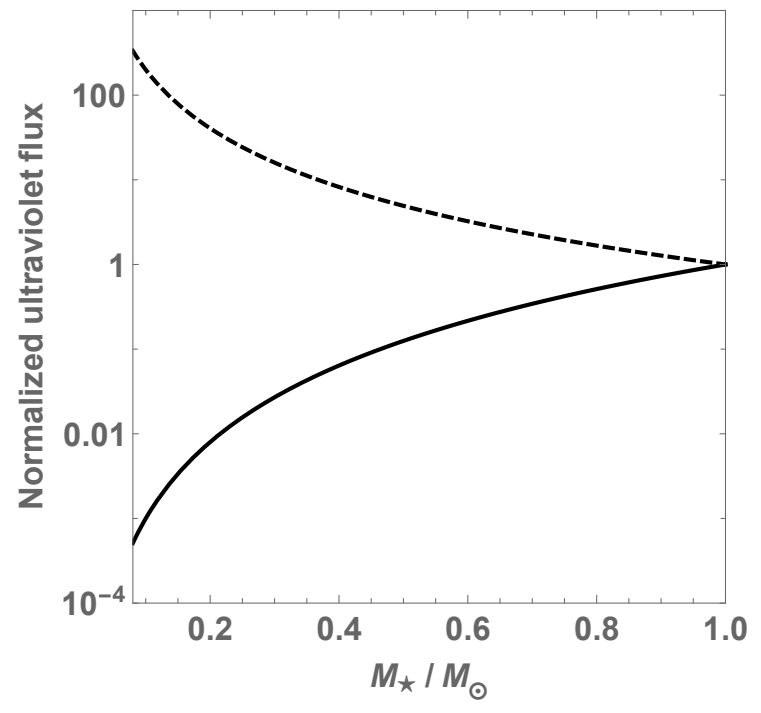

FIG. 1 Schematic representation of the UV fluxes received by Earth-analogs around low-mass stars normalized with respect to the Earth as a function of the stellar mass $M_{\star}$ (in units of solar mass $\left.M_{\odot}\right)$. The solid curve denotes the flux of bioactive UV radiation $(\sim 200-400 \mathrm{~nm})$, whereas the dashed curve denotes the flux of Ly $\alpha$ radiation. The former may contribute to the origin of life, driving evolutionary innovations and speciation, DNA damage and inhibiting photosynthesis. The latter is believed to play a key role in the photolysis of molecules such as $\mathrm{H}_{2} \mathrm{O}$ and $\mathrm{CO}_{2}$, thus enabling water depletion and the build-up of atmospheric $\mathrm{O}_{2}$. Scaling relations have been adopted from Lingam and Loeb (2018c).

velocity of $v_{\text {esc }}=\sqrt{2 G M_{p} / R_{p}}$, then we have $\frac{1}{2} \dot{M}_{p} v_{\text {esc }}^{2} \sim$ $\pi \mathcal{F}_{E U V} R_{p}^{2}$. From this relation, we obtain

$$
\dot{M}_{p}=\frac{\eta_{E U V}}{K_{\mathrm{eff}}} \frac{\pi R_{p}^{3} \mathcal{F}_{E U V}}{G M_{p}}
$$

where $\eta_{E U V}$ is the heating efficiency factor, while $K_{\text {eff }}$ is a parameter of order unity that encapsulates the effect of stellar tidal forces (Erkaev et al., 2007). In the above expression, we note that the EUV flux can also be replaced by the XUV flux $(0.6-120 \mathrm{~nm})$ that includes the contribution from X-rays (Ribas et al., 2016). This formula has been derived based on the assumption of energy balance, but other regimes are also conceivable (Owen, 2019); for instance, photon number limitation (as opposed to energy limitation) may regulate the atmospheric escape rate (Owen and Alvarez, 2016).

All stars go through a pre-main-sequence (PMS) phase, during which their luminosity is fuelled mostly by gravitational contraction as opposed to the nuclear fusion of hydrogen (McKee and Ostriker, 2007). The importance of this phase, particularly for low-mass stars, stems from the fact that the luminosity during the PMS phase can be as much as two orders of magnitude higher compared to the point where the star enters the main-sequence (Baraffe et al., 2002). Moreover, for very low-mass stars (with $M_{\star} \lesssim 0.1 M_{\odot}$ ), the PMS phase lasts for a few Gyr, whereas it is $\lesssim 0.1$ Gyr for solar-type stars (Ramirez and Kaltenegger, 2014). Thus, the collective effect of the PMS phase is that planets around low-mass stars that would otherwise be situated within the HZ are not located in it as a result of the enhanced luminosity. In other words, these planets exceed the runaway greenhouse threshold, thereby leading to significant losses of water (Ramirez and Kaltenegger, 2014; Luger and Barnes, 2015; Bolmont et al., 2017; Tian et al., 2018).

The basic expectation is that the water vapor in the atmosphere injected via the greenhouse effect would undergo photolysis to yield hydrogen and oxygen. As the former has a much lower mass, it is more susceptible to atmospheric escape. This can be verified by converting $\dot{M}_{p}$ in (10) from $\mathrm{kg} / \mathrm{yr}$ to moles/yr, as it yields an inverse dependence on the mass of the chemical species. Hence, the hydrogen would be lost to space, leaving behind massive $\mathrm{O}_{2}$ atmospheres. In reality, the situation is more complex and would depend on factors such as the 
EUV or XUV flux $\left(\mathcal{F}_{X U V}\right)$, the planet's mass $\left(M_{p}\right)$ and water inventory (Tian, 2015b). The rate of atmospheric $\mathrm{O}_{2}$ buildup $\left(\dot{P}_{\mathrm{O}_{2}}\right)$ was analytically estimated by Luger and Barnes (2015), and is expressible as

$$
\dot{P}_{O_{2}} \sim 0.138 \text { bars } / \operatorname{Myr}\left(\frac{\mathcal{F}_{X U V}}{\mathcal{F}_{\oplus}}\right)\left(\frac{R}{R_{\oplus}}\right)^{-1}\left(\frac{\eta_{X U V}}{0.30}\right)
$$

where $\eta_{X U V}$ denotes the XUV absorption efficiency, Myr $\equiv 10^{6}$ yr and $\mathcal{F}_{\oplus} \approx 4.6 \times 10^{-3} \mathrm{~J} \mathrm{~m}^{-2} \mathrm{~s}^{-1}$ is the XUV flux incident on Earth. This result can be rewritten in terms of $R_{p}$ by applying the mass-radius relationship $M_{p} \propto R_{p}^{3.7}$ for rocky planets (Zeng et al., 2016). However, when $\mathcal{F}_{X U V}$ exceeds a certain threshold, $\mathrm{O}_{2}$ is "dragged" along with hydrogen (due to the emergence of strong hydrodynamic flows) and is therefore subject to escape. The corresponding threshold is given by

$$
\mathcal{F}_{c} \sim 0.18 \mathrm{~J} \mathrm{~m}^{-2} \mathrm{~s}^{-1}\left(\frac{M_{p}}{M_{\oplus}}\right)^{2}\left(\frac{R_{p}}{R_{\oplus}}\right)^{-3}\left(\frac{\eta_{X U V}}{0.30}\right)^{-1} .
$$

Hence, in the regime $\mathcal{F}_{X U V} \geq \mathcal{F}_{c}$, the rate of atmospheric $\mathrm{O}_{2}$ buildup undergoes saturation and does not depend on $\mathcal{F}_{X U V}$ (Luger and Barnes, 2015). The corresponding value of $\dot{P}_{\mathrm{O}_{2}}$ for $\mathcal{F}_{X U V} \geq \mathcal{F}_{c}$ becomes

$$
\dot{P}_{O_{2}} \sim 5.35 \text { bar/Myr }\left(\frac{M_{p}}{M_{\oplus}}\right)^{2}\left(\frac{R_{p}}{R_{\oplus}}\right)^{-4} .
$$

As noted above, $M_{p}$ can be eliminated by utilizing the mass-radius relationship for terrestrial planets. In comparison, the rate of $\mathrm{O}_{2}$ contributed to Earth's atmosphere indirectly by photosynthesis (entailing the burial of organic matter) is $\sim 0.06$ bars/Myr (Holland, 2002). If (11) or (13) exceeds the atmospheric $\mathrm{O}_{2}$ sinks for Earth-like planets, oxygen can build up in the atmosphere and produce an ozone layer (Catling and Kasting, 2017a).

A number of studies have been conducted that pertain to the production of abiotic $\mathrm{O}_{2}$ and $\mathrm{O}_{3}$ in the atmosphere due to the photolysis of $\mathrm{H}_{2} \mathrm{O}$ and $\mathrm{CO}_{2}$ by XUV radiation (Segura et al., 2007; Wordsworth and Pierrehumbert, 2013, 2014; Tian et al., 2014; Harman et al., 2015; Narita et al., 2015; Gao et al., 2015). Hence, the detection of $\mathrm{O}_{2}$ and $\mathrm{O}_{3}$ at high levels in exoplanetary atmospheres opens up the possibility of a "false positive", corresponding to the apparent detection of a biosignature despite it not being produced by biological activity. Several methods have been proposed for distinguishing abiotic $\mathrm{O}_{2}$ from that generated by biological sources (Meadows, 2017; Meadows et al., 2018b).

As the net effect of UV photolysis of water leads to the depletion of oceans, this has important ramifications for habitability since liquid water is one of the requirements for life. Before discussing this point further, some of the studies pertaining to water loss from Proxima $b$ and the TRAPPIST-1 planets are worth mentioning. In the case of Proxima b, it has been predicted that $<1 M_{o c, \oplus}$ has been lost over its history (Ribas et al., 2016), where $M_{o c, \oplus} \approx 1.4 \times 10^{21} \mathrm{~kg}$ is the mass of Earth's oceans today. This could, however, still leave behind an $\mathrm{O}_{2}$ atmosphere of $\sim 100$ bar. In the case of the TRAPPIST-1 planets, the innermost two planets (TRAPPIST-1b and TRAPPIST-1c) may have lost as much as $15 M_{o c, \oplus}$ (Bolmont et al., 2017) but this value could drop below $1 M_{o c, \oplus}$ for the outer planets (Bourrier et al., 2017).

One of the Earth's unique features is that the fraction of the surface covered by land (0.3) is comparable to that covered by water (0.7). However, in the majority of cases, we can expect one of two possible scenarios to arise, especially around low-mass stars. First, the initial water inventory, which depends on the physical mechanisms involved in the delivery of $\mathrm{H}_{2} \mathrm{O}$ during terrestrial planet formation (O'Brien et al., 2018), may vary widely (Raymond et al., 2007; Mulders et al., 2015b; Raymond and Izidoro, 2017). Hence, there will be many worlds with water inventories much higher than the Earth - some of the planets in the TRAPPIST-1 system are an excellent example, as their water inventory could exceed that of the Earth by 2 orders of magnitude (Grimm et al., 2018; Unterborn et al., 2018a,b; Dorn et al., 2018). These worlds are expected to comprise only oceans on the surface, thereby lacking landmasses altogether, and both observational (Rogers, 2015; Wolfgang and Lopez, 2015; Chen and Kipping, 2017; Jin and Mordasini, 2018; Lozovsky et al., 2018) and theoretical (Alibert and Benz, 2017; Simpson, 2017) studies indicate they are potentially quite common. The habitability of these "water worlds" (Kuchner, 2003; Léger et al., 2004) has been the subject of many studies over the past few years (Noack et al., 2017; Kite and Ford, 2018; Ramirez and Levi, 2018).

On the other hand, as we have seen previously, XUV radiation during the long PMS phase of low-mass stars is very effective at depleting several oceans worth of water. Moreover, the deep-water cycle transports water from the oceans to the underlying mantle (Hirschmann, 2006); the water reservoir in the latter may exceed the former by up to an order of magnitude on Earth (Korenaga, 2008; Ni et al., 2017). In many cases, worlds with a moderate initial water inventory could end up being desiccated and eventually transformed into desert planets (Abe et al., 2011; Zsom et al., 2013). In contrast, for certain waterrich worlds, XUV radiation may actually play a positive role by removing excess water and ensuring that landmasses are exposed on the surface. As per these arguments, we predict the following two features for planets in the HZ of low-mass stars: (a) worlds with Earth-like water inventories are rare, and (b) the water inventory is describable by a bimodal distribution (water- or desertworlds). Both of these points appear to be consistent with numerical simulations carried out by Tian and Ida (2015); see also Zain et al. (2018) for a related analysis of solar-type stars.

This aspect poses major issues for habitability for the 
following reasons. If the worlds are covered almost entirely by land, water availability becomes an issue and the resultant arid environments are expected to have low biomass densities (Hadley and Szarek, 1981). On the other hand, if the surface consists exclusively of oceans, the availability of bioessential elements like phosphorus - widely accepted as the ultimate limiting nutrient for the biological productivity of oceans (Tyrrell, 1999) - becomes problematic because it is primarily delivered via rivers and the atmosphere through continental weathering (Paytan and McLaughlin, 2007), implying that the absence of continents will lower the influx of dissolved phosphorus by a few orders of magnitude provided that the oceans are alkaline and water-rock interactions exist at the seafloor (Lingam and Loeb, 2019b, 2018d).

A recent analysis of the surface water fraction $\left(f_{w}\right)$ proposed that $0.3<f_{w}<0.9$ is optimal for the buildup of $\mathrm{O}_{2}$ in the atmosphere (Lingam and Loeb, 2019c), which represented a major evolutionary event. As the fraction of Earth-sized worlds in the $\mathrm{HZ}$ with water inventories that lie within this range is presumably small $(\lesssim 1 \%)$ see Tian and Ida (2015) and Lingam and Loeb (2019c) it seems plausible that Earth-like worlds with an admixture of landmasses and oceans are not very common.

\section{B. Origin of life}

The issue of how and where life originated remains one of science's most profound and enduring mysteries. The reader may consult McCollom (2013); Luisi (2016); Sutherland (2016) for recent overviews of this subject. Currently, life uses deoxyribonucleic acid (DNA) for the storage and transmission of genetic information, while proteins, among several other functions, play the role of catalysts. In the $1980 \mathrm{~s}$, it was discovered that ribonucleic acid (RNA) was capable of carrying information as well as facilitating catalysis. This gave impetus to the idea, which was first proposed in the 1960s, that life on Earth began with self-replicating RNA molecules. The hypothesis came to be subsequently known as the RNA world (Gilbert, 1986), and has witnessed rapid advancements within the last decade (Joyce, 2002; Orgel, 2004; Neveu et al., 2013; Higgs and Lehman, 2015).

Before proceeding further, it is crucial to recognize that the RNA world is not the only hypothesis for the origin of life (abiogenesis). A number of models have proposed that self-sustaining metabolic networks arose prior to the evolution of replicators like RNA (Wächtershäuser, 2007; Ruiz-Mirazo et al., 2014; Goldford and Segrè, 2018). The geochemical environment in which life originated has also subject to numerous investigations (Stüeken et al., 2013; Kitadai and Maruyama, 2018). A number of hypotheses have advocated that life arose in land-based environ- ments such as tidal pools (Romer, 1933; Lathe, 2004), ${ }^{12}$ intermountain valleys (Benner et al., 2012), hot springs and geysers (Mulkidjanian et al., 2012). In contrast, one of the most prominent hypotheses suggests that life originated in alkaline hydrothermal vents situated at the ocean floor (Martin et al., 2008; Sojo et al., 2016).

Many of the surficial geochemical environments customarily feature a wide assortment of minerals, which are believed to have played a crucial role in the emergence and early evolution of life (Cleaves et al., 2012; Hazen, 2017). However, it should be recognized that most of the land-based environments in this category are not anticipated to be widely prevalent on water worlds, which were introduced in Sec. III.A. On the other hand, life's origin in environments at the ocean floor (e.g. hydrothermal vents) remains a distinct possibility unless the water content is high enough to drive the formation of highpressure ices, thereby cutting off direct water-rock interactions (Noack et al., 2016).

Broadly speaking, there are three broad classes of macromolecules (apart from carbohydrates) that are necessary for "life as we know it". The first is nucleic acids (DNA and RNA), which serve as repositories for genetic information. The second is proteins, as they catalyze biochemical reactions as well as playing a vital role in maintaining cell structure and enabling cell signaling. The third is lipids, which form an integral part of cell membranes and also facilitate energy storage. The building blocks of nucleic acids are nucleotides - for example, RNA is synthesized from the polymerization of ribonucleotides - whereas proteins are polymers of amino acids.

Ever since the 1960s and 1970s, it has been appreciated that UV radiation enabled the synthesis of prebiotic compounds such as amino acids (Sagan and Khare, 1971). However, it is only within the last decade that the putative significance of UV radiation has been appreciated in greater detail owing to a number of recent developments outlined next (Sutherland, 2017).

- Studies have demonstrated that UV light accords a selective advantage to RNA-like molecules (Gustavsson et al., 2010; Šponer et al., 2016), implying that it may play a crucial role in enabling their polymerization (Dibrova et al., 2012).

- Several laboratory experiments display a tendency to form complex organic mixtures ("tar") that essentially represent a dead end insofar as abiogenesis is concerned. This issue is known as the "asphalt problem", but it might be bypassed in certain geochemical environments with UV radiation playing a potentially important role (Benner et al., 2012).

12 The biological ramifications of tidal modulations arising from the presence of two planetary companions were discussed by Balbus (2014) and Lingam and Loeb (2018e). 
- The synthesis of biomolecular building blocks is very challenging, especially without regular manual intervention and under conditions that ostensibly resemble that of early Earth. Remarkably, several notable breakthroughs have been achieved recently that rely on UV light. The organic compounds synthesized include: (i) RNA components, e.g. ribonucleotides (Powner et al., 2009; Islam and Powner, 2017), (ii) simple sugar-related molecules (Ritson and Sutherland, 2012, 2013; Todd et al., 2018), (iii) precursors of nucleic acids, amino acids, lipids and carbohydrates from interlinked pathways involving plausible feedstock molecules such as hydrogen cyanide (Patel et al., 2015; Xu et al., 2018), and (iv) iron-sulfur clusters, which play a vital role in metabolism (Bonfio et al., 2017).

- RNA nucleotides are stable to irradiation by UV photons, which has been used to argue that they originated in the high-UV environments on early Earth (Rios and Tor, 2013; Beckstead et al., 2016; Ranjan and Sasselov, 2016).

Thus, taken collectively, there is compelling, but not necessarily definitive, evidence that UV light played an important role in the origin of life on the planetary surface.

This brings us to the question of which planets in the $\mathrm{HZ}$ receive higher fluxes of quiescent (i.e., background) UV radiation. Several studies indicate planets in the HZ of M-dwarfs receive bioactive UV fluxes (with $200<\lambda<$ $400 \mathrm{~nm}$ ) at the surface that are around 100-1000 times lower compared to early Earth (Rugheimer et al., 2015a; Ranjan et al., 2017); see also Buccino et al. (2007). It must also be appreciated that the UV flux reaching the surface depends on a number of planetary characteristics such as the atmospheric composition and column density. As the bioactive UV flux depends on stellar properties, the region around the host star where the planet receives enough UV photons for prebiotic chemistry (the UV zone) will not always overlap with the classical HZ.

This issue was investigated in Guo et al. (2010), where it was concluded that the UV zone lies inward of the HZ when $T_{\text {eff }}<4600 \mathrm{~K}$. A more recent analysis, based on the latest prebiotic pathways for synthesizing RNA, protein and lipid precursors (Patel et al., 2015; Xu et al., 2018), found that stars with $T_{\text {eff }}<4400 \mathrm{~K}$ were not likely to drive the formation of these building blocks if the potential role of stellar flares (see Sec. IV) is neglected (Rimmer et al., 2018). In addition to the stellar temperature, which serves as a rough proxy for the stellar mass, metallicity, i.e., abundance of elements other than $\mathrm{H}$ or $\mathrm{He}$, can also influence the location of the UV zone (Oishi and Kamaya, 2016) and its overlap with the HZ.

While these studies deal with the spatial overlap between the UV zone and the HZ, it must be appreciated that the rates of these prebiotic reactions may also depend on the bioactive UV fluxes (Ranjan et al., 2017).
Hence, planets in the HZ of M-dwarfs could require much longer timescales for abiogenesis. This fact was utilized by Lingam and Loeb (2018c) to suggest that the timescale for abiogenesis $\left(t_{A}\right)$ is

$$
t_{A} \sim t_{A, \oplus}\left(\frac{M_{\star}}{M_{\odot}}\right)^{-\kappa},
$$

where $\kappa \approx 3$ for $M_{\star} \lesssim M_{\odot}, \kappa \approx 1$ for $M_{\star} \gtrsim M_{\odot}$ and $t_{A, \oplus}$ is the timescale for the origin of life on Earth, which has a strict upper bound of 0.8 Gyr. Coupled to the fact that Earth-analogs around M-dwarfs are expected to lose their atmospheres more rapidly, it was proposed that stars with $M_{\star} \lesssim 0.4 M_{\odot}$ are relatively unlikely to host biospheres (Lingam and Loeb, 2019a).

\section{Evolution of complex life}

We will briefly discuss how stellar radiation, particularly in the UV and visible ranges, influences the trajectories of biological evolution.

\section{Biological damage due to UV radiation}

It is well-known that UV radiation, particularly in the range $180<\lambda<300 \mathrm{~nm}$, is capable of suppressing photosynthesis and causing damage to DNA and other biomolecules (Voet et al., 1963; Sagan, 1973; Teramura and Sullivan, 1994; Cadet et al., 2005). Yet, on the other hand, it must be recognized that UV radiation might potentially function as a selection agent, a driver of evolutionary innovations and speciation (Sagan, 1973; Rothschild, 1999; Evans and Gaston, 2005).

As this topic has been the subject of many investigations, we will concern ourselves with describing only a couple of recent examples. Before embarking on that discussion, we note that a number of environments effectively shield organisms from the damaging effects of UV radiation such as a layer of soil, ocean (Cleaves and Miller, 1998), hazes in the atmosphere (Arney et al., 2016), and other screening compounds (Cockell and Knowland, 1999). Another possibility is that complex evolutionary adaptations, such as those evinced by a wide array of microbes (Gao and Garcia-Pichel, 2011; Gabani and Singh, 2013; Pacelli et al., 2017; Jung et al., 2017), could protect organisms from high doses of UV radiation.

The biologically effective irradiance (BEI) was computed for Earth-analogs around different stars in Rugheimer et al. (2015a). The BEI can be envisioned as the product of the surface UV flux and the DNA action spectrum, where the latter quantifies the extent of DNA damage at different wavelengths. Two broad conclusions can be immediately drawn. First, the BEI for very low-mass stars (with $M_{\star} \lesssim 0.1 M_{\odot}$ ) is always about $\gtrsim 100$ times smaller than the Earth at the same epoch. 
Second, the presence of an ozone layer, not surprisingly, plays a major role in determining the BEI. For example, it was estimated that the BEI at 3.9 Gyr ago on Earth was about 600 times the present-day value.

A similar study was undertaken by O'Malley-James and Kaltenegger (2017), who concluded that two other parameters which influence the BEI are the stellar activity and the atmospheric pressure along with the presence (or absence) of an ozone layer. It was found that planets around more active stars, with partially eroded atmospheres, are likely to receive high UV doses at the surface. The issue of rarefied atmospheres is particularly relevant, since we have seen earlier that stellar winds and UV radiation can drive atmospheric escape and erosion. However, even in the worst-case scenario, it was found that a combination of evolutionary adaptations and ecological niches are theoretically capable of sustaining life (O'Malley-James and Kaltenegger, 2019).

\section{Photosynthesis}

On Earth, most life is dependent, either directly or indirectly, upon photosynthesis. The radiation typically employed by photoautotrophs (photosynthetic organisms) ranges between wavelengths of 400 and $700 \mathrm{~nm}$ (Hohmann-Marriott and Blankenship, 2011), and goes by the name of photosynthetically active radiation (PAR). If we approximate the stellar spectrum as black body radiation, Wien's displacement law implies

$$
\lambda_{\max } T_{\mathrm{eff}}=2.9 \times 10^{-3} \mathrm{~m} \cdot \mathrm{K},
$$

where $\lambda_{\max }$ is the wavelength at which the black body spectrum peaks. Choosing $T_{\text {eff }} \approx 5778 \mathrm{~K}$ for the Sun yields $\lambda_{\max } \approx 500 \mathrm{~nm}$, which sits almost in the middle of PAR range. In qualitative terms, this makes sense because photoautotrophs may have evolved to optimize the number of photons that they collect for synthesizing organic compounds (Kiang et al., 2007a).

Estimating the PAR range for other stars, especially M-dwarfs, is very difficult since it depends on the spectral properties of the host star, the atmospheric composition and the pigments that enable photosynthesis (Kiang et al., 2007a; Bains et al., 2014). One of the most striking features of vegetation on Earth is the "red edge", i.e. an increase in the reflectance by roughly an order of magnitude at $\sim 700 \mathrm{~nm}$. The importance of the red edge stems from the fact that it represents a viable biosignature that could be detectable with future telescopes (Seager et al., 2005; Kaltenegger, 2017). Owing to the aforementioned issues, it is not easy to predict where the analog of red edge would exist for M-dwarfs. It does, however, seem plausible that the edge would be shifted to near-infrared wavelengths around $1.1 \mu \mathrm{m}$ (Kiang et al., 2007b); see, however, Takizawa et al. (2017). Other methods for detecting signatures of photosynthesis include distinctive features in circularly and linearly polarized spectra (Sparks et al., 2009; Berdyugina et al., 2016) and angledependent reflectivity (Doughty and Wolf, 2010).

If we restrict our attention to the same PAR as on Earth, we are confronted with a potential problem. As low-mass stars tend to radiate primarily in the infrared, the availability of photons in the PAR range will be reduced. This raises the question of whether photosynthesis on planets around M-dwarfs is feasible. As most of the photosynthesis on Earth leads to the production of $\mathrm{O}_{2}$ (Fischer et al., 2016), many studies have concentrated on this particular process. The simplified reaction presented below illustrates the formation of organic matter and oxygen via oxygenic photosynthesis.

$$
\mathrm{CO}_{2}+2 \mathrm{H}_{2} \mathrm{O}+h \nu \rightarrow \mathrm{CH}_{2} \mathrm{O}+\mathrm{H}_{2} \mathrm{O}+\mathrm{O}_{2} .
$$

It was argued by Gale and Wandel (2017) that oxygenic photosynthesis in the PAR is feasible on planets around low-mass stars, primarily because the side facing the star (assuming synchronous rotation) will receive continuous illumination that compensates for the moderate photon flux. A detailed analysis of the prospects for photosynthesis on Proxima b was undertaken by Ritchie et al. (2018) - the rate of synthesis of organic compounds via photosynthesis was estimated to be $\sim 17 \%$ that of the Earth despite the photon flux in the PAR range being only $3 \%$ of the Earth. However, it was also argued therein that oxygenic photosynthesis might not evolve on planets around M-dwarfs as this process accords minimal competitive advantage. Lehmer et al. (2018) investigated whether enough photons would be available to support an Earth-like biosphere on M-dwarf exoplanets, and found that many of them are incapable of doing so; see also Lingam and Loeb (2019d). In particular, if the maximum wavelength of PAR is specified to be $750 \mathrm{~nm}$, none of the TRAPPIST-1 planets in the HZ appear to have the capacity for sustaining Earth-like biospheres. Relatively sparse biospheres due to the limited PAR fluxes may also have the additional disadvantage of producing weak $\mathrm{O}_{2}$ signals that are not easily detectable.

\section{Oxygen and complex life}

There are sufficient grounds to conclude that the buildup of $\mathrm{O}_{2}$ in the atmosphere may have constituted a rate-limiting step insofar the development of complex life is concerned on other worlds (Knoll, 1985; O'Malley and Powell, 2016; Judson, 2017). Yet, it should also be recognized that similar environmental conditions and evolutionary trajectories for complex life need not prevail on all worlds. The development of the ozone layer and the expansion of aerobic metabolism, which releases about an order of magnitude more energy compared to its anaerobic counterparts (Koch and Britton, 2008), are two ex- 
amples of the profound changes triggered by the rise of $\mathrm{O}_{2}$. The oxygen levels in Earth's atmosphere as a function of geological time were subject to fluctuations, but there is compelling evidence that the $\mathrm{O}_{2}$ levels increased from a very small value to roughly $1 \%$ of the present atmospheric level (PAL) around $\sim 2.4 \mathrm{Ga}$ (Gumsley et al., 2017; Knoll and Nowak, 2017). Many different mechanisms have been advanced to explain this rise in oxygen, known as the Great Oxygenation Event (GOE), such as changes in volcanism and the oxidation state of continents (Kasting, 2013; Lyons et al., 2014).

One of the chief hypotheses for the GOE that we focus on concerns the production of $\mathrm{O}_{2}$ in the atmosphere through UV photolysis (Catling et al., 2001; Catling and Kasting, 2017b). Organic matter is produced from oxygenic photosynthesis via (16), and this is subsequently decomposed by other microbes to release methane $\left(\mathrm{CH}_{4}\right)$. Methane undergoes UV photolysis with $\mathrm{H}$ escaping to space and the carbon combining with $\mathrm{O}_{2}$ to form $\mathrm{CO}_{2}$. The net reaction is expressible as follows:

$$
2 \mathrm{H}_{2} \mathrm{O}+\text { "biosphere" }+h \nu \rightarrow \mathrm{O}_{2}+4 \mathrm{H}(\uparrow \text { space }) .
$$

The key point is that this reaction does not represent the abiotic photolysis of water since reactions mediated by the biosphere are necessary. In addition, as we have seen in Sec. III.A, there are several abiotic pathways by which $\mathrm{O}_{2}$ is produced through UV photolysis on planets, particularly those around low-mass stars. These mechanisms could enable the build-up of $\mathrm{O}_{2}$ at rates much faster than those driven by biological factors.

It is therefore conceivable that the time required for $\mathrm{O}_{2}$ levels to reach a certain value (e.g. 1\% PAL) via (17) is much shorter for planets that receive high UV fluxes at the appropriate wavelengths. An important feature of Mdwarfs is that their ratio of far-UV $(117-175 \mathrm{~nm})$ to nearUV $(175-320 \mathrm{~nm})$ fluxes are $\sim 1000$ times higher than the corresponding ratio for the Sun (Tian et al., 2014), because of enhanced UV emission from the chromosphere and transition regions (Linsky et al., 2013). The Ly $\alpha$ flux $\left(\Phi_{L}\right)$, which is responsible for a large fraction of water and methane photolysis, for Earth-analogs can be approximated by a power law of the form $\Phi_{L} \propto M_{\star}^{\zeta}$, where $\zeta \approx-2.3$ for $M_{\star} \lesssim M_{\odot}$ and $\zeta \approx 3.3$ for $M_{\star} \gtrsim M_{\odot}$ (Lingam and Loeb, 2018c). Note that the flux for stars more massive than the Sun is higher as a larger fraction of the black body radiation is emitted in the UV regime.

Hence, if the oxygenation time $\left(t_{O_{2}}\right)$ scales inversely with $\Phi_{L}$, the evolution of complex aerobic life from microbial anaerobic organisms may require a shorter amount of time on Earth-analogs orbiting stars that have a higher mass than the Sun (Livio, 1999), although these stars are less abundant with respect to solar-mass stars.

\section{STELLAR FLARES}

Stellar flares are explosive phenomena on the stellar surface that lead to the release of energy in various forms such as electromagnetic radiation, plasma and energetic particles (Benz, 2017). The central engine behind this energy release is believed to be magnetic reconnection (Priest and Forbes, 2002), which entails changes in magnetic topology through the breaking and reconnection of field lines, thus resulting in the rapid conversion of magnetic energy into other forms of energy (Biskamp, 2000). Classical magnetic reconnection models, which were originally developed in the 1950s, result in energy release over long timescales that are not supported by observations, but subsequent developments in this area have achieved much progress in terms of addressing this issue (Priest, 2014; Shibata and Magara, 2011; Comisso et al., 2016).

The largest flares documented in modern history have energies of $\sim 10^{25} \mathrm{~J}$, with the largest one on record being the Carrington event from 1859 (Carrington, 1859) that released a total energy of approximately $5 \times 10^{25}$ $\mathrm{J}$ (Cliver and Dietrich, 2013). However, it is important to appreciate that flares with energies $\geq 10^{26} \mathrm{~J}$, known as superflares, are also feasible on theoretical and observational grounds. The Kepler mission has recorded a wealth of observational data regarding the statistics of superflares (Maehara et al., 2012; Shibayama et al., 2013; Davenport, 2016; Namekata et al., 2017; Notsu et al., 2019). For solar-type stars with similar rotation rates as the Sun, it has been found that

$$
\frac{d N}{d E} \propto E^{-\alpha},
$$

with $\alpha \approx 1.5-2$, where $N(E)$ represents the occurrence rate of superflares and $E$ denotes the energy of these superflares (Maehara et al., 2012, 2015; Günther et al., 2019). The power-law exponent is close to that documented for normal flares on the Sun (Hannah et al., 2011). The maximum amount of energy that may be released, in theory, during a flare event is expressible as (Shibata et al., 2013):

$$
E \sim 10^{28} \mathrm{~J}\left(\frac{\epsilon}{0.1}\right)\left(\frac{B_{A}}{0.1 \mathrm{~T}}\right)^{2}\left(\frac{f_{A}}{0.3}\right)^{3 / 2}\left(\frac{R_{\star}}{R_{\odot}}\right)^{3} .
$$

Note that $R_{\star}$ and $R_{\odot}$ are the stellar and solar radii respectively, whereas $\epsilon$ represents the "efficiency" of converting magnetic energy into flare energy. In this formula, $B_{A}$ denotes the magnetic field strength in the active region(s), and $f_{A}$ is the fraction of the star's surface that is covered by the active region(s). The active regions constitute the sites of solar flares and other stellar activity, and sunspots serve as indicators of active regions.

In the case of the Sun, it has been theorized that flares of $\sim 10^{27} \mathrm{~J}$ can occur over a timescale of $\sim 2000 \mathrm{yrs}$ (Shibayama et al., 2013), but this is hard to verify on 
account of relatively sparse direct evidence. The studies by Miyake et al. (2012) and Miyake et al. (2013), which discovered rapid variations in the concentrations of radionuclides in tree rings might be indicative of superflare activity. The extreme upper limit on the maximum flare energy released from Sun-like stars appears to be $\sim 10^{30}$ J (Schrijver et al., 2012; Lingam and Loeb, 2017b), although it remains highly uncertain as to whether such superflares could actually arise in reality.

In our subsequent discussion, we will mostly concentrate on planets in the $\mathrm{HZ}$ of M-dwarfs as these stars are known to be very active and typically, but not always, produce flares and superflares at rates higher than G-type stars like the Sun (Scalo et al., 2007; Maehara et al., 2012). Both TRAPPIST-1 and Proxima Centauri have been documented to produce flares regularly - the number of flares with energies $\gtrsim 10^{26} \mathrm{~J}$ is predicted to be $\sim 10^{-2}$ per day based on flare statistics (Davenport et al., 2016; Vida et al., 2017; Howard et al., 2018); see also MacGregor et al. (2018). Recall that these planets are situated much closer to their host stars, may have weak magnetic fields and could be subjected to rapid atmospheric erosion from stellar winds.

\section{A. Electromagnetic radiation}

It has long been appreciated that flares lead to enhanced UV fluxes at the surfaces of planets within the HZ of M-dwarfs (Heath et al., 1999; Smith et al., 2004a). In the majority of instances, this has been viewed as a negative strike against the habitability of M-dwarf exoplanets, despite the fact that many UV shielding mechanisms are feasible, as described in Sec. III.C.1. Nonetheless, we shall highlight a couple of recent studies that illustrate the detrimental effects before discussing the potential positives later.

In an important study, Segura et al. (2010) simulated the fluxes of UV radiation that were delivered to the surface of an Earth-analog at a distance of 0.16 AU orbiting the star $\mathrm{AD}$ Leonis $\left(M_{\star} \approx 0.4 M_{\odot}\right.$ and age $\left.<0.3 \mathrm{Gyr}\right)$ during a flare with a total energy of $\sim 10^{27} \mathrm{~J}$. It was found that the amount of UV flux increased by a factor of around 50 at the peak of the flare compared to the quiescent phase prior to the onset of the flare. Although this was seemingly a significant increase, most of the UV enhancement was in the UV-A (315-400 nm) range, which has a much weaker effect on damaging DNA (by a factor of $\sim 100$ ) compared to shorter UV wavelengths. However, an important aspect worth appreciating is that this result presumed the existence of ozone. Taking ozone depletion into account (see Sec. IV.C), the same flare was estimated to produce higher UV-A and UV-B (280-315 $\mathrm{nm}$ ) fluxes at the peak of the flare.

Estrela and Valio (2018) analyzed how a hypothetical Earth-analog at 1 AU around the solar-analog Kepler-
96 (with $M_{\star} \approx M_{\odot}$ ) would respond to a superflare with energy $E \sim 1.8 \times 10^{28} \mathrm{~J}$. This led to an enhancement in the UV flux by nearly two orders of magnitude, and the BEI (see Sec. III.C.1) was found to exceed even that of radiation-resistant extremophile Deinococcus radiodurans in the absence of ozone. However, the presence of an ozone layer or an ocean depth of $\sim 12 \mathrm{~m}$ would suffice to protect microbes akin to $D$. radiodurans from the UV radiation emitted during superflares.

Moving on to the positive effects, we have noted in Sec. III.B that planets around M-dwarfs may be characterized by a paucity of bioactive UV radiation. However, it is possible, in principle, for prebiotic reactions to take place during the flaring phase because of the enhanced UV fluxes, while remaining inactive during the quiescent phase (Ranjan et al., 2017; Buccino et al., 2007). This hypothesis requires further experimental tests before it can be confirmed or invalidated. Based on the available flare statistics, Rimmer et al. (2018) proposed that $\sim 20 \%$ of M-dwarfs are sufficiently active so as to provide sufficient UV fluxes for synthesizing the precursors of biomolecules.

In the same spirit, we saw in Sec. III.C.2 that Earthlike biospheres based on photosynthesis may not be sustainable on low-mass stars. However, an important point worth recognizing is that flares can also deliver photons in the PAR range. Hence, when the effects of stellar flares are included, theoretical calculations seem to indicate that the PAR flux may be raised by approximately one order of magnitude (Mullan and Bais, 2018) (see, however, Lingam and Loeb 2019d), although the averaged photosynthetic efficiency of planets in the $\mathrm{HZ}$ of M-dwarfs is still anticipated to be lower than that of the Earth (Scharf, 2019). Finally, the high fluxes of UV radiation incident on the surface during flaring events are expected to result in intermittently enhanced mutation rates, thereby serving as agents of ecological and evolutionary change (Smith et al., 2004b).

\section{B. Coronal mass ejections}

Coronal mass ejections (CMEs) are large loads of plasma and magnetic fields expelled from the host star. They are generally, but not always, linked with flares (Webb and Howard, 2012). The majority of CMEs carry a mass of $\lesssim 10^{13} \mathrm{~kg}$ and move at velocities of order 100 $1000 \mathrm{~km} / \mathrm{s}$. One of the important reasons for studying CMEs is that they can facilitate the acceleration of energetic particles through shock waves (Kilpua et al., 2017), which is addressed in Sec. IV.C. We will, instead, focus on their effects on planetary magnetospheres and atmospheric erosion.

To begin with, it is instructive to consider the parameters of a sizable CME. A CME with parameters commensurate with the famous Carrington event is predicted to have a density that is $\sim 50$ times higher than the current 
solar wind density and a velocity that is $\sim 4$ times greater (Ngwira et al., 2014). Thus, based on the preceding discussion in Sec. II.A, we find that the corresponding ram pressure would be $\sim 800$ times higher than that of the present-day solar wind. Substituting this result into (2), we find that the magnetopause distance for an Earthanalog would be compressed to roughly one-third of its steady-state value for the current solar wind.

From (3), we see that the mass-loss rate should be enhanced by a factor of $\sim 200$, and utilizing (6) reveals that the atmospheric escape rate should increase by a factor of $\sim 200$ with respect to the steady-state as well. This prediction is in good agreement with numerical simulations that yield an enhancement of $\sim 110$ (Dong et al., 2017b). Observational evidence from the ongoing Mars Atmosphere and Volatile Evolution (MAVEN) mission has also revealed that CMEs (smaller than Carringtontype events) can result in the enhancement of Martian atmospheric ion escape rates by roughly an order of magnitude (Dong et al., 2015; Jakosky et al., 2015).

Although the above discussion pertains to our Solar system, we observe that these two implications also apply to extrasolar systems. For instance, owing to a combination of the weak planetary magnetic fields, close-in distances and frequency of CME impacts (Kay et al., 2016), the effects of CMEs are expected to significantly compress the magnetospheres of M-dwarf exoplanets (Khodachenko et al., 2007) and enhance the escape rates leading to cumulative atmospheric losses of $\sim 10-100$ bars (Lammer et al., 2007). At the same time, we wish to underscore the fact that the mass-loss rates from active stars, especially M-dwarfs, due to CMEs is not tightly constrained. This is because of the fact that most studies rely on extrapolations based on putative correlations between the X-ray fluences of flares and the kinetic energies and masses of CMEs, although the extent to which such extrapolations are valid remains unknown (Odert et al., 2017). The presence of large-scale magnetic fields may, for instance, impose upper limits on the kinetic energy of CMEs (Alvarado-Gómez et al., 2018).

Bearing these caveats in mind, we note that the massloss rates due to CMEs from magnetically active stars may be 1-3 orders of magnitude higher than the current mass carried away by the steady-state solar wind today (Drake et al., 2013; Cranmer, 2017). Hence, from (6), it also follows that the atmospheric escape rates will be enhanced by the same degree for active (usually young) stars. In other words, the timescale for the total depletion of the atmosphere computed in Sec. II.B could represent an upper bound. The magnetospheres will also be compressed by a factor of a few owing to the enhanced value of the ram pressure in (2).

Thus, when viewed cumulatively, it seems plausible that CMEs exacerbate the issues discussed in the context of stellar winds in Sec. II.

\section{Stellar proton events}

The majority of large stellar flares are accompanied by the production of stellar/solar energetic particles (SEPs), and these phenomena are known as stellar/solar proton events (SPEs). The mechanisms behind their production are complex, but can be divided into two broad categories: "impulsive" events involving magnetic reconnection and "gradual" events involving fast shock waves propelled by CMEs (Reames, 2013). Of the two, it is the latter that produce higher fluences of SEPs (Desai and Giacalone, 2016), and are therefore expected to be more prominent. The kinetic energies of SEPs (mostly protons or electrons) are usually in the $\mathrm{keV}$ and $\mathrm{MeV}$ ranges, but the maximum values can reach several $\mathrm{GeV}$ (Mewaldt, 2006). As direct measurements of extrasolar SEP fluences are not currently feasible, most models rely on extrapolations from empirical scalings (Takahashi et al., 2016; Youngblood et al., 2017). It must, however, be appreciated that these predictions may break down at high SEP fluences (Hudson, 2015; Usoskin, 2017).

It has been duly recognized, since nearly half a century ago, that SEPs lead to the formation of hydrogen and nitrogen oxides (Crutzen, 1979; López-Puertas et al., 2005). The latter, in particular, have been shown to facilitate the depletion of ozone (Solomon, 1999) through catalytic reactions, of which one of them is

$$
\mathrm{NO}+\mathrm{O}_{3} \rightarrow \mathrm{NO}_{2}+\mathrm{O}_{2}
$$

where $\mathrm{NO}$ and $\mathrm{NO}_{2}$ are nitric oxide and nitrogen dioxide, respectively (Crutzen et al., 1975). Hence, highfluence SPEs can lead to the destruction of the ozone layer (Tilley et al., 2019), which in turn results in high doses of UV radiation reaching the surface. In principle, this might be offset by the presence of high concentrations of $\mathrm{N}_{2} \mathrm{O}$ (which absorb UV radiation) (Rugheimer et al., 2013, 2015b). As noted previously, high-UV radiation fluxes do not preclude the existence of life under a layer of water or in subsurface environments, but they may impose constraints on surface habitability.

Segura et al. (2010) analyzed how a putative SPE associated with the AD Leonis flare, discussed earlier in Sec. IV.A, affected the ozone layer. It was found that the ozone depletion reached a maximum of $94 \%$ provided that the Earth-analog was unmagnetized. Very recently, a superflare with energy $\sim 10^{26.5} \mathrm{~J}$ was detected from Proxima Centauri (Howard et al., 2018). In the presence of an ozone layer, the effects on habitability were not significant. However, when the effects of SPEs were taken into account, it was found that $90 \%$ of the ozone layer could be lost within five years. In this scenario, the amount of UV-C radiation $(<280 \mathrm{~nm})$ that reached the surface was almost two orders of magnitude higher than the critical flux that would kill $90 \%$ of a given population of the radiation-resistant microbe $D$. radiodurans. 
A similar study was undertaken to assess the impact of repeated SPEs on a hypothetical Earth-analog around the star GJ1243, and it was found that ozone depletion of $94 \%$ could occur in as little as 10 yrs (Tilley et al., 2019). The net result of all these studies appears to be that complete ozone depletion might take place within $\mathcal{O}\left(10^{5}\right)$ yrs, although it must be recognized that this timescale depends on the flaring frequency, planetary magnetic field and atmospheric composition; see also Tabataba-Vakili et al. (2016) for a discussion of how SPEs impact M-dwarf exoplanets. Consequently, it would result in a quiescent UV-C flux that may lie above the tolerance threshold of $D$. radiodurans; see, however, O'Malley-James and Kaltenegger (2019). This issue will probably be exacerbated by subsequent flares that increase the UV-C flux by 2-3 orders of magnitude.

By examining the available empirical evidence for the correlations between flare energy, the fluence of SEPs, and the degree of ozone depletion, Lingam and Loeb (2017b) presented a phenomenological relation between the ozone depletion $\left(\mathcal{D}_{\mathrm{O}_{3}}\right)$ and the flare energy:

$$
\mathcal{D}_{O_{3}} \sim 2.8 \%\left(\frac{E}{10^{25} \mathrm{~J}}\right)^{9 / 25}\left(\frac{a_{p}}{1 \mathrm{AU}}\right)^{-18 / 25} .
$$

Hence, it can be verified that a flare with $E \sim 2 \times 10^{29} \mathrm{~J}$ would lead to complete ozone depletion $\left(\mathcal{D}_{O_{3}} \approx 100 \%\right)$ for a terrestrial planet at $1 \mathrm{AU}$, whereas the corresponding value at $0.1 \mathrm{AU}$ is $E \sim 2 \times 10^{27} \mathrm{~J}$. If we make use of the relations $a_{p} \propto L_{\star}^{1 / 2} \propto M_{\star}^{7 / 4}$ (Böhm-Vitense, 1992) for Earth-analogs, we obtain $\mathcal{D}_{O_{3}} \propto E^{0.36} M_{\star}^{-1.26}$.

Setting aside the issue of ozone depletion, another problematic issue arising from SEPs is that their impact with atmospheres induces the formation of secondary particle cascades that increase the radiation dose received at the surface (Melott and Thomas, 2011; Atri and Melott, 2014). This topic was recently studied by Atri (2017) for a wide range of planetary parameters. In general, it was found that the radiation dose at the surface increased when: (a) the atmospheric column density was lowered, (b) the flare energy was increased, (c) the planetary magnetic moment was reduced, and (d) the star-planet distance was lowered. In the case of exoplanets around M-dwarfs, it is conceivable that one or more of (a)-(d) are applicable. While this does not rule out the prospects for life, the critical radiation doses for macroscopic multicellular organisms on Earth (e.g. mammals) are exceeded under certain circumstances.

However, there are potential benefits associated with SPEs as well. To begin with, the abiotic synthesis of the building blocks of life requires suitable energy sources and pathways (Miller and Urey, 1959; Chyba and Sagan, 1992; Deamer and Weber, 2010). One of the most important feedstock molecules for prebiotic synthesis is hydrogen cyanide (Sutherland, 2016). Detailed numerical simulations carried out by Airapetian et al. (2016) demonstrate that hydrogen cyanide (HCN) can be synthesized at concentrations of tens of parts per million by volume in the lower atmosphere during SPEs. The production of the aforementioned nitrogen oxides via SEPs can also be associated with a positive component: these compounds are attractive electron acceptors that may facilitate the emergence of metabolic pathways and life at alkaline hydrothermal vents (Wong et al., 2017) or shallow ponds and lakes (Ranjan et al., 2019).

Looking beyond feedstock molecules like $\mathrm{HCN}$ and formaldehyde $\left(\mathrm{CH}_{2} \mathrm{O}\right)$, laboratory experiments have demonstrated that a wide array of biomolecular building blocks such as amino acids and nucleobases, which are essential components of nucleotides (i.e., monomers of nucleic acids), are synthesized with relatively high efficiency when gasesous mixtures are irradiated by highenergy protons (Dartnell, 2011; Kobayashi et al., 1998; Miyakawa et al., 2002). The averaged energy flux $\left(\Phi_{\mathrm{SEP}}\right)$ delivered to the surface of a planet with a 1-bar atmosphere via SEPs is (Lingam et al., 2018):

$$
\Phi_{\mathrm{SEP}} \sim 50 \mathrm{~J} \mathrm{~m}^{-2} \mathrm{yr}^{-1}\left(\frac{\dot{N}}{1 \text { day }^{-1}}\right)\left(\frac{a_{p}}{1 \mathrm{AU}}\right)^{-2},
$$

where $\dot{N}$ denotes the number of "large" SPEs expected to impact the planet per day, which could reach a maximum of order unity for young Sun-like stars as well as M-dwarfs (Kay et al., 2016). It is evident from (22) that M-dwarf exoplanets will receive higher SEP fluxes due to the smaller values of $a_{p}$ (Fraschetti et al., 2019). In turn, this could result in the enhanced synthesis of prebiotic compounds (Nava-Sedeño et al., 2016), thereby partially offsetting the deficiency of UV light delineated in Sec. III.B. By drawing upon laboratory experiments, which are admittedly idealized, Lingam et al. (2018) suggested that the production rates of organics were given by

$$
\begin{aligned}
& \dot{\mathcal{M}}_{A} \sim 10^{7} \mathrm{~kg} / \mathrm{yr}\left(\frac{\Phi_{\mathrm{SEP}}}{100 \mathrm{~J} \mathrm{~m}^{-2} \mathrm{yr}^{-1}}\right)\left(\frac{R_{p}}{R_{\oplus}}\right)^{2}, \\
& \dot{\mathcal{M}}_{N} \sim 10^{4} \mathrm{~kg} / \mathrm{yr}\left(\frac{\Phi_{\mathrm{SEP}}}{100 \mathrm{~J} \mathrm{~m}^{-2} \mathrm{yr}^{-1}}\right)\left(\frac{R_{p}}{R_{\oplus}}\right)^{2},
\end{aligned}
$$

where $\dot{\mathcal{M}}_{A}$ and $\dot{\mathcal{M}}_{N}$ denoted the rate of production of amino acids and nucleobases, respectively. On early Earth, it was found that $\dot{\mathcal{M}}_{A}$ was comparable to the production rate of amino acids from electrical discharges (lightning), and 3-4 orders of magnitude higher than the delivery rate of these compounds through meteorites.

From a geological standpoint, the primordial Earth possibly required a high concentration of greenhouse gases to prevent its oceans from freezing due to the lower luminosity of the Sun ( $70 \%$ of the present-day value) during this epoch (Sagan and Mullen, 1972). This has led to many proposals to explain how temperatures above freezing were achieved (Feulner, 2012). Nitrous oxide $\left(\mathrm{N}_{2} \mathrm{O}\right)$ is efficiently formed during SPEs (Airapetian 
et al., 2016), ${ }^{13}$ and has a greenhouse potential that is $\sim 300$ times higher than $\mathrm{CO}_{2}$ over a 100 -yr period (Voigt et al., 2017). The latter feature lends credence to the idea that it may have contributed to the greenhouse warming of early Earth (Canfield et al., 2010) and possibly other habitable planets. Signatures of $\mathrm{N}_{2} \mathrm{O}$ and biosignature gases like $\mathrm{O}_{2}$ could be "highlighted" during episodes of magnetic activity, thereby making them more detectable (Airapetian et al., 2017b).

\section{DISCUSSION}

M-dwarfs like Proxima Centauri and TRAPPIST-1 are $\lesssim 10$ times more abundant than the Sun (Chabrier, 2003; Robles et al., 2008) and have stellar lifetimes that are 100-1000 times greater (Tarter et al., 2007; Adams and Laughlin, 1997; Loeb et al., 2016). Furthermore, exoplanets around these stars are easier to detect and their atmospheres can be analyzed via transit spectroscopy (Winn, 2010; Fujii et al., 2018), thus enabling the ready detection of biomarkers. Hence, in light of these facts, we are confronted with the following question: how is exoplanetary habitability influenced by the properties of the host star? One possible answer is that physical mechanisms could act in concert to suppress the likelihood of life's emergence on M-dwarf exoplanets relative to their counterparts around solar-type stars.

In this Colloquium, we have encountered a number of potential reasons as to why the habitability of M-dwarf exoplanets might be reduced. These include atmospheric erosion due to intense stellar winds, coronal mass ejections and UV radiation, paucity of photons for the origin of life and photosynthesis, and an inhospitable surface environment due to radiation from stellar flares and solar proton events. Yet, it is equally vital to appreciate that numerous nonlinear feedback mechanisms are at play, and that none of the aforementioned factors rule out the prospects for life altogether.

For instance, exoplanets around M-dwarfs could have started out with massive hydrogen-helium atmospheres and lost them via atmospheric erosion involving UV radiation, stellar winds or active phases of supermassive black holes to yield potentially habitable worlds (Luger et al., 2015; Chen et al., 2018). It is also possible that planets started with high water inventories that were depleted through UV photolysis and hydrogen escape to yield worlds with a mixture of land and oceans on the surface. In addition, planets may have formed outside the $\mathrm{HZ}$ and escaped the brunt of the long and intense pre-main-sequence phase of M-dwarfs, before eventually migrating inwards into the $\mathrm{HZ}$ at a later stage; see

13 Moreover, it may also build up on planets with low-UV fluxes as the latter would otherwise destroy $\mathrm{N}_{2} \mathrm{O}$ (Rugheimer et al., 2013).
Tamayo et al. (2017) and Ormel et al. (2017). Finally, M-dwarf planetary systems might possess inherent advantages such as the enhanced transport of life between planets via lithopanspermia by several orders of magnitude compared to the Earth-Mars system (Steffen and Li, 2016; Lingam and Loeb, 2017c; Krijt et al., 2017). However, in each of the above instances, either a high degree of fine tuning might be required or the feasibility of the proposed mechanisms remains indeterminate.

The question posed earlier is important from a practical standpoint because of the fact that resources such as the observation time, technological capabilities of current facilities and funding are all limited. Hence, the selection of the most optimal target stars and planets when searching for biosignatures will be of the utmost importance in the future, when we are confronted with a diverse array of planetary systems around different stars (Horner and Jones, 2010; Kaltenegger et al., 2010; Lingam and Loeb, 2018a). In this respect, the importance of theoretical modeling as a tool for identifying which targets merit the highest consideration becomes manifest. Moreover, theoretical models can also help us figure out what kind of hypothetical biospheres may exist and what to potentially expect when searching for signatures of life.

The ultimate answer to this question will, of course, necessitate detailed observations that are free of preconceived biases. With the upcoming launch of the James Webb Space Telescope, the ongoing development of ground-based extremely large telescopes, and mission concepts for future space telescopes, the characterization of terrestrial planets and searching for biosignatures is expected to become feasible in the upcoming decades (Fujii et al., 2018; Rodler and López-Morales, 2014; Snellen et al., 2015; Barstow and Irwin, 2016; Morley et al., 2017; Kiang et al., 2018; Madhusudhan, 2019). These developments might not only help up answer the age-old question of whether we are alone but also assess the rarity or commonality of life in the Universe.

\section{ACKNOWLEDGMENTS}

We thank the reviewers for their perspicacious and detailed reports which were very valuable for improving the paper. This work was supported in part by the Breakthrough Prize Foundation, Harvard University's Faculty of Arts and Sciences, and the Institute for Theory and Computation (ITC) at Harvard University.

\section{REFERENCES}

C. Bailey, in The Stoic and Epicurean Philosophers, edited by W. J. Oates (The Modern Library, 1957) pp. 3-68. M. Mayor and D. Queloz, Nature 378, 355 (1995).

M. A. C. Perryman, Rep. Prog. Phys. 63, 1209 (2000).

N. M. Batalha, Proc. Natl. Acad. Sci. USA 111, 12647 (2014). 
W. J. Borucki, Rep. Prog. Phys. 79, 036901 (2016).

J. N. Winn and D. C. Fabrycky, Annu. Rev. Astron. Astrophys. 53, 409 (2015).

M. Perryman, The Exoplanet Handbook, 2nd ed. (Cambridge University Press, 2018).

R. Heller, D. Williams, D. Kipping, M. A. Limbach, E. Turner, R. Greenberg, T. Sasaki, É. Bolmont, O. Grasset, K. Lewis, R. Barnes, and J. I. Zuluaga, Astrobiology 14, 798 (2014).

A. Teachey and D. M. Kipping, Sci. Adv. 4, eaav1784 (2018).

P. Ball, Chem. Rev. 108, 74 (2008).

S. A. Benner, A. Ricardo, and M. A. Carrigan, Curr. Opin. Chem. Biol. 8, 672 (2004).

W. Bains, Astrobiology 4, 137 (2004).

D. Schulze-Makuch and L. N. Irwin, Life in the Universe: Expectations and Constraints, 2nd ed. (Springer-Verlag, 2008).

A. G. Hayes, Annu. Rev. Earth Planet. Sci. 44, 57 (2016).

J. L. Mitchell and J. M. Lora, Annu. Rev. Earth Planet. Sci. 44, 353 (2016).

B. M. Jakosky, M. Slipski, M. Benna, P. Mahaffy, M. Elrod, R. Yelle, S. Stone, and N. Alsaeed, Science 355, 1408 (2017).

C. Dong, Y. Lee, Y. Ma, M. Lingam, S. Bougher, J. Luhmann, S. Curry, G. Toth, A. Nagy, V. Tenishev, X. Fang, D. Mitchell, D. Brain, and B. Jakosky, Astrophys. J. Lett. 859, L14 (2018a).

G. Gonzalez, Orig. Life Evol. Biosph. 35, 555 (2005).

J. F. Kasting, D. P. Whitmire, and R. T. Reynolds, Icarus 101, 108 (1993).

R. K. Kopparapu, R. Ramirez, J. F. Kasting, V. Eymet, T. D. Robinson, S. Mahadevan, R. C. Terrien, S. DomagalGoldman, V. Meadows, and R. Deshpande, Astrophys. J. 765, 131 (2013).

R. K. Kopparapu, R. M. Ramirez, J. SchottelKotte, J. F. Kasting, S. Domagal-Goldman, and V. Eymet, Astrophys. J. Lett. 787, L29 (2014).

J. Yang, G. Boué, D. C. Fabrycky, and D. S. Abbot, Astrophys. J. Lett. 787, L2 (2014).

A. Zsom, Astrophys. J. 813, 9 (2015).

R. k. Kopparapu, E. T. Wolf, J. Haqq-Misra, J. Yang, J. F. Kasting, V. Meadows, R. Terrien, and S. Mahadevan, Astrophys. J. 819, 84 (2016).

J. Haqq-Misra, R. K. Kopparapu, N. E. Batalha, C. E. Harman, and J. F. Kasting, Astrophys. J. 827, 120 (2016).

R. M. Ramirez, Geosciences 8, 280 (2018).

E. W. Schwieterman, C. T. Reinhard, S. L. Olson, C. E. Harman, and T. W. Lyons, Astrophys. J. 878, 19 (2018).

V. Dobos, R. Heller, and E. L. Turner, Astron. Astrophys. 601, A91 (2017).

S. R. Kane and N. R. Hinkel, Astrophys. J. 762, 7 (2013).

M. Cuntz, Astrophys. J. 780, 14 (2014).

T. M. Hoehler, J. P. Amend, and E. L. Shock, Astrobiology 7, 819 (2007).

H. Morowitz and E. Smith, Complexity 13, 51 (2007).

E. Branscomb, T. Biancalani, N. Goldenfeld, and M. Russell, Phys. Rep. 677, 1 (2017).

W. B. Moore, A. Lenardic, A. M. Jellinek, C. L. Johnson, C. Goldblatt, and R. D. Lorenz, Nat. Astron. 1, 0043 (2017).

E. Tasker, J. Tan, K. Heng, S. Kane, D. Spiegel, R. Brasser, A. Casey, S. Desch, C. Dorn, J. Hernlund, C. Houser, M. Laneuville, M. Lasbleis, A.-S. Libert, L. Noack, C. Unterborn, and J. Wicks, Nat. Astron. 1, 0042 (2017).
S. H. Dole, Habitable planets for man (Blaisdell Pub. Co., 1964).

H. Lammer, J. H. Bredehöft, A. Coustenis, M. L. Khodachenko, L. Kaltenegger, O. Grasset, D. Prieur, F. Raulin, P. Ehrenfreund, M. Yamauchi, J.-E. Wahlund, J.-M. Grießmeier, G. Stangl, C. S. Cockell, Y. N. Kulikov, J. L. Grenfell, and H. Rauer, Astron. Astrophys. Rev. 17, 181 (2009).

E. J. Javaux and V. Dehant, Astron. Astrophys. Rev. 18, 383 (2010).

C. H. Lineweaver and A. Chopra, Annu. Rev. Earth Planet. Sci. 40, 597 (2012).

S. Seager, Science 340, 577 (2013).

C. S. Cockell, T. Bush, C. Bryce, S. Direito, M. FoxPowell, J. P. Harrison, H. Lammer, H. Landenmark, J. Martin-Torres, N. Nicholson, L. Noack, J. O'MalleyJames, S. J. Payler, A. Rushby, T. Samuels, P. Schwendner, J. Wadsworth, and M. P. Zorzano, Astrobiology 16, 89 (2016).

B. L. Ehlmann, F. S. Anderson, J. Andrews-Hanna, D. C. Catling, P. R. Christensen, B. A. Cohen, C. D. Dressing, C. S. Edwards, L. T. Elkins-Tanton, K. A. Farley, C. I. Fassett, W. W. Fischer, A. A. Fraeman, M. P. Golombek, V. E. Hamilton, A. G. Hayes, C. D. K. Herd, B. Horgan, R. Hu, B. M. Jakosky, J. R. Johnson, J. F. Kasting, L. Kerber, K. M. Kinch, E. S. Kite, H. A. Knutson, J. I. Lunine, P. R. Mahaffy, N. Mangold, F. M. McCubbin, J. F. Mustard, P. B. Niles, C. Quantin-Nataf, M. S. Rice, K. M. Stack, D. J. Stevenson, S. T. Stewart, M. J. Toplis, T. Usui, B. P. Weiss, S. C. Werner, R. D. Wordsworth, J. J. Wray, R. A. Yingst, Y. L. Yung, and K. J. Zahnle, J. Geophys. Res. E 121, 1927 (2016).

J. Horner and B. W. Jones, Int. J. Astrobiol. 9, 273 (2010).

L. Kaltenegger, C. Eiroa, I. Ribas, F. Paresce, M. Leitzinger, P. Odert, A. Hanslmeier, M. Fridlund, H. Lammer, C. Beichman, W. Danchi, T. Henning, T. Herbst, A. Léger, R. Liseau, J. Lunine, A. Penny, A. Quirrenbach, H. Röttgering, F. Selsis, J. Schneider, D. Stam, G. Tinetti, and G. J. White, Astrobiology 10, 103 (2010).

M. Lingam and A. Loeb, Astrophys. J. Lett. 857, L17 (2018a). G. Anglada-Escudé, P. J. Amado, J. Barnes, Z. M. Berdiñas, R. P. Butler, G. A. L. Coleman, I. de La Cueva, S. Dreizler, M. Endl, B. Giesers, S. V. Jeffers, J. S. Jenkins, H. R. A. Jones, M. Kiraga, M. Kürster, M. J. LópezGonzález, C. J. Marvin, N. Morales, J. Morin, R. P. Nelson, J. L. Ortiz, A. Ofir, S.-J. Paardekooper, A. Reiners, E. Rodríguez, C. Rodríguez-López, L. F. Sarmiento, J. P. Strachan, Y. Tsapras, M. Tuomi, and M. Zechmeister, Nature 536, 437 (2016).

M. Gillon, E. Jehin, S. M. Lederer, L. Delrez, J. de Wit, A. Burdanov, V. Van Grootel, A. J. Burgasser, A. H. M. J. Triaud, C. Opitom, B.-O. Demory, D. K. Sahu, D. Bardalez Gagliuffi, P. Magain, and D. Queloz, Nature 533, 221 (2016).

M. Gillon, A. H. M. J. Triaud, B.-O. Demory, E. Jehin, E. Agol, K. M. Deck, S. M. Lederer, J. de Wit, A. Burdanov, J. G. Ingalls, E. Bolmont, J. Leconte, S. N. Raymond, F. Selsis, M. Turbet, K. Barkaoui, A. Burgasser, M. R. Burleigh, S. J. Carey, A. Chaushev, C. M. Copperwheat, L. Delrez, C. S. Fernandes, D. L. Holdsworth, E. J. Kotze, V. Van Grootel, Y. Almleaky, Z. Benkhaldoun, P. Magain, and D. Queloz, Nature 542, 456 (2017).

L. Delrez, M. Gillon, A. H. M. J. Triaud, B.-O. Demory, J. de Wit, J. G. Ingalls, E. Agol, E. Bolmont, A. Burdanov, A. J. 
Burgasser, S. J. Carey, E. Jehin, J. Leconte, S. Lederer, D. Queloz, F. Selsis, and V. Van Grootel, Mon. Not. R. Astron. Soc. 475, 3577 (2018).

S. L. Grimm, B.-O. Demory, M. Gillon, C. Dorn, E. Agol, A. Burdanov, L. Delrez, M. Sestovic, A. H. M. J. Triaud, M. Turbet, E. Bolmont, A. Caldas, J. d. Wit, E. Jehin, J. Leconte, S. N. Raymond, V. V. Grootel, A. J. Burgasser, S. Carey, D. Fabrycky, K. Heng, D. M. Hernandez, J. G. Ingalls, S. Lederer, F. Selsis, and D. Queloz, Astron. Astrophys. 613, A68 (2018).

J. A. Dittmann, J. M. Irwin, D. Charbonneau, X. Bonfils, N. Astudillo-Defru, R. D. Haywood, Z. K. BertaThompson, E. R. Newton, J. E. Rodriguez, J. G. Winters, T.-G. Tan, J.-M. Almenara, F. Bouchy, X. Delfosse, T. Forveille, C. Lovis, F. Murgas, F. Pepe, N. C. Santos, S. Udry, A. Wünsche, G. A. Esquerdo, D. W. Latham, and C. D. Dressing, Nature 544, 333 (2017).

J. Laskar, F. Joutel, and P. Robutel, Nature 361, 615 (1993).

J. Kasting, How to Find a Habitable Planet (Princeton University Press, 2010).

R. T. Pierrehumbert, Principles of Planetary Climate (Cambridge University Press, 2010).

D. C. Catling and J. F. Kasting, Atmospheric Evolution on Inhabited and Lifeless Worlds (Cambridge University Press, 2017).

M. Turbet, J. Leconte, F. Selsis, E. Bolmont, F. Forget, I. Ribas, S. N. Raymond, and G. Anglada-Escudé, Astron. Astrophys. 596, A112 (2016).

E. T. Wolf, Astrophys. J. Lett. 839, L1 (2017).

I. A. Boutle, N. J. Mayne, B. Drummond, J. Manners, J. Goyal, F. Hugo Lambert, D. M. Acreman, and P. D. Earnshaw, Astron. Astrophys. 601, A120 (2017).

T. Alberti, V. Carbone, F. Lepreti, and A. Vecchio, Astrophys. J. 844, 19 (2017).

M. Turbet, E. Bolmont, J. Leconte, F. Forget, F. Selsis, G. Tobie, A. Caldas, J. Naar, and M. Gillon, Astron. Astrophys. 612, A86 (2018).

V. S. Meadows, G. N. Arney, E. W. Schwieterman, J. LustigYaeger, A. P. Lincowski, T. Robinson, S. D. DomagalGoldman, R. Deitrick, R. K. Barnes, D. P. Fleming, R. Luger, P. E. Driscoll, T. R. Quinn, and D. Crisp, Astrobiology 18, 133 (2018a).

A. P. Lincowski, V. S. Meadows, D. Crisp, T. D. Robinson, R. Luger, J. Lustig-Yaeger, and G. N. Arney, Astrophys. J. 867, 76 (2018).

J. L. Sarmiento and N. Gruber, Ocean Biogeochemical Dynamics (Princeton University Press, 2006).

W. H. Schlesinger and E. S. Bernhardt, Biogeochemistry: An Analysis of Global Change, 3rd ed. (Academic Press, 2013).

G. Gonzalez, D. Brownlee, and P. Ward, Icarus 152, 185 (2001).

C. H. Lineweaver, Y. Fenner, and B. K. Gibson, Science 303, 59 (2004).

N. Prantzos, Space Sci. Rev. 135, 313 (2008).

M. G. Gowanlock and I. S. Morrison, "The Habitability of our Evolving Galaxy," in Habitability of the Universe Before Earth, Astrobiology: Exploring Life on Earth and Beyond, Vol. 1, edited by P. H. Rampelotto, J. Seckbach, and R. Gordon (Academic Press, 2018) pp. 149-171.

S. E. Thorsett, Astrophys. J. Lett. 444, L53 (1995).

A. L. Melott and B. C. Thomas, Astrobiology 11, 343 (2011).

T. Piran and R. Jimenez, Phys. Rev. Lett. 113, 231102 (2014).
D. Sloan, R. Alves Batista, and A. Loeb, Sci. Rep. 7, 5419 (2017).

A. Balbi and F. Tombesi, Sci. Rep. 7, 16626 (2017).

J. C. Forbes and A. Loeb, Mon. Not. R. Astron. Soc. 479, 171 (2018).

M. Lingam, I. Ginsburg, and S. Bialy, Astrophys. J. 877, 62 (2019).

J. C. Tarter, P. R. Backus, R. L. Mancinelli, J. M. Aurnou, D. E. Backman, G. S. Basri, A. P. Boss, A. Clarke, D. Deming, L. R. Doyle, E. D. Feigelson, F. Freund, D. H. Grinspoon, R. M. Haberle, S. A. Hauck, II, M. J. Heath, T. J. Henry, J. L. Hollingsworth, M. M. Joshi, S. Kilston, M. C. Liu, E. Meikle, I. N. Reid, L. J. Rothschild, J. Scalo, A. Segura, C. M. Tang, J. M. Tiedje, M. C. Turnbull, L. M. Walkowicz, A. L. Weber, and R. E. Young, Astrobiology 7, 30 (2007).

J. Scalo, L. Kaltenegger, A. G. Segura, M. Fridlund, I. Ribas, Y. N. Kulikov, J. L. Grenfell, H. Rauer, P. Odert, M. Leitzinger, F. Selsis, M. L. Khodachenko, C. Eiroa, J. Kasting, and H. Lammer, Astrobiology 7, 85 (2007).

A. L. Shields, S. Ballard, and J. A. Johnson, Phys. Rep. 663, 1 (2016).

G. Chabrier and I. Baraffe, Annu. Rev. Astron. Astrophys. 38, 337 (2000).

G. Chabrier and I. Baraffe, Astron. Astrophys. 327, 1039 (1997).

K. G. Stassun, L. Hebb, K. Covey, A. A. West, J. Irwin, R. Jackson, M. Jardine, J. Morin, D. Mullan, and I. N. Reid, in 16th Cambridge Workshop on Cool Stars, Stellar Systems, and the Sun, Astronomical Society of the Pacific Conference Series, Vol. 448, edited by C. Johns-Krull, M. K. Browning, and A. A. West (2011) pp. 505-516.

F. C. Adams and G. Laughlin, Rev. Mod. Phys. 69, 337 (1997).

G. Chabrier, Publ. Astron. Soc. Pac. 115, 763 (2003).

X. Bonfils, X. Delfosse, S. Udry, T. Forveille, M. Mayor, C. Perrier, F. Bouchy, M. Gillon, C. Lovis, F. Pepe, D. Queloz, N. C. Santos, D. Ségransan, and J.-L. Bertaux, Astron. Astrophys. 549, A109 (2013).

C. D. Dressing and D. Charbonneau, Astrophys. J. 807, 45 (2015).

G. D. Mulders, I. Pascucci, and D. Apai, Astrophys. J. 798, $112(2015 \mathrm{a})$.

J. N. Winn, "Exoplanet Transits and Occultations," in Exoplanets, edited by S. Seager (University of Arizona Press, 2010) pp. 55-77.

Y. Fujii, D. Angerhausen, R. Deitrick, S. Domagal-Goldman, J. L. Grenfell, Y. Hori, S. R. Kane, E. Pallé, H. Rauer, N. Siegler, K. Stapelfeldt, and K. B. Stevenson, Astrobiology 18, 739 (2018).

E. N. Parker, Astrophys. J. 128, 664 (1958).

E. Priest, Magnetohydrodynamics of the Sun (Cambridge University Press, 2014).

O. Cohen, J. J. Drake, A. Glocer, C. Garraffo, K. Poppenhaeger, J. M. Bell, A. J. Ridley, and T. I. Gombosi, Astrophys. J. 790, 57 (2014).

O. Cohen, A. Glocer, C. Garraffo, J. J. Drake, and J. M. Bell, Astrophys. J. Lett. 856, L11 (2018).

W. Baumjohann and R. A. Treumann, Basic Space Plasma Physics (Imperial College Press, 2012).

T. I. Gombosi, Physics of the Space Environment, Cambridge Atmospheric and Space Science Series (Cambridge University Press, 1998). 
M. G. Kivelson and C. T. Russell, Introduction to Space Physics (Cambridge University Press, 1995).

C. Garraffo, J. J. Drake, and O. Cohen, Astrophys. J. Lett. 833, L4 (2016).

C. Garraffo, J. J. Drake, O. Cohen, J. D. Alvarado-Gómez, and S. P. Moschou, Astrophys. J. Lett. 843, L33 (2017).

C. Dong, M. Jin, M. Lingam, V. S. Airapetian, Y. Ma, and B. van der Holst, Proc. Natl. Acad. Sci. USA 115, 260 (2018b).

R. Fitzpatrick, Plasma Physics: An Introduction (CRC Press, 2014).

B. J. Wargelin and J. J. Drake, Astrophys. J. 578, 503 (2002).

B. E. Wood, J. L. Linsky, H.-R. Müller, and G. P. Zank, Astrophys. J. Lett. 547, L49 (2001).

C. Dong, M. Lingam, Y. Ma, and O. Cohen, Astrophys. J. Lett. 837, L26 (2017a).

A. Reiners and G. Basri, Astron. Astrophys. 496, 787 (2009).

J. Morin, J.-F. Donati, P. Petit, X. Delfosse, T. Forveille, and M. M. Jardine, Mon. Not. R. Astron. Soc. 407, 2269 (2010).

A. Reiners, Living Rev. Sol. Phys. 9, 1 (2012).

A. A. Vidotto, M. Jardine, J. Morin, J.-F. Donati, P. Lang, and A. J. B. Russell, Astron. Astrophys. 557, A67 (2013).

V. See, M. Jardine, A. A. Vidotto, P. Petit, S. C. Marsden, S. V. Jeffers, and J. D. do Nascimento, Astron. Astrophys. 570, A99 (2014).

J.-M. Grießmeier, A. Stadelmann, U. Motschmann, N. K. Belisheva, H. Lammer, and H. K. Biernat, Astrobiology 5, 587 (2005).

M. López-Morales, N. Gómez-Pérez, and T. Ruedas, Orig. Life Evol. Biosph. 41, 533 (2011).

U. R. Christensen, Space Sci. Rev. 152, 565 (2010).

J.-M. Grießmeier, in Characterizing Stellar and Exoplanetary Environments, Astrophysics and Space Science Library, Vol. 411, edited by H. Lammer and M. Khodachenko (2015) pp. 213-237.

J. Leconte, H. Wu, K. Menou, and N. Murray, Science 347, $632(2015)$.

V. V. Makarov, Astrophys. J. 810, 12 (2015).

A. M. Vinson and B. M. S. Hansen, Mon. Not. R. Astron. Soc. 472, 3217 (2017).

E. Bolmont, S. N. Raymond, and J. Leconte, Astron. Astrophys. 535, A94 (2011).

R. Barnes, Celest. Mech. Dyn. Astron. 129, 509 (2017).

J. I. Zuluaga, S. Bustamante, P. A. Cuartas, and J. H. Hoyos, Astrophys. J. 770, 23 (2013).

C. Dong, J. G. Luhmann, Y. Ma, M. Lingam, A. Bhattacharjee, Y. Lee, S. W. Bougher, L. Wang, A. Glocer, R. J. Strangeway, S. Curry, X. Fang, G. Toth, A. F. Nagy, R. J. Lillis, D. L. Mitchell, D. Brain, and B. M. Jakosky, AGU Fall Meeting Abstracts P31C-3736 (2018c).

E. G. Blackman and J. A. Tarduno, Mon. Not. R. Astron. Soc. 481, 5146 (2018).

H. Gunell, R. Maggiolo, H. Nilsson, G. Stenberg Wieser, R. Slapak, J. Lindkvist, M. Hamrin, and J. De Keyser, Astron. Astrophys. 614, L3 (2018).

M. Lingam, Astrophys. J. Lett. 874, L28 (2019).

W. I. Axford, J. Geophys. Res. 73, 6855 (1968).

R. Schunk and A. Nagy, Ionospheres: Physics, Plasma Physics, and Chemistry, Cambridge Atmospheric and Space Science Series (Cambridge Univ. Press, 2009).

S. Sakai, K. Seki, N. Terada, H. Shinagawa, T. Tanaka, and Y. Ebihara, Geophys. Res. Lett. 45, 9336 (2018).

L. R. Dartnell, Astrobiology 11, 551 (2011).
J. E. Horvath and D. Galante, Int. J. Astrobiol. 11, 279 (2012).

J.-M. Grießmeier, A. Stadelmann, J. L. Grenfell, H. Lammer, and U. Motschmann, Icarus 199, 526 (2009).

D. Atri, B. Hariharan, and J.-M. Grießmeier, Astrobiology 13, 910 (2013).

J.-M. Grießmeier, F. Tabataba-Vakili, A. Stadelmann, J. L. Grenfell, and D. Atri, Astron. Astrophys. 587, A159 (2016).

J. L. Grenfell, Phys. Rep. 713, 1 (2017).

F. Tian, Annu. Rev. Earth Planet. Sci. 43, 459 (2015a).

H. Lammer, J. F. Kasting, E. Chassefière, R. E. Johnson, Y. N. Kulikov, and F. Tian, Space Sci. Rev. 139, 399 (2008).

D. A. Brain, F. Bagenal, Y.-J. Ma, H. Nilsson, and G. Stenberg Wieser, J. Geophys. Res. E 121, 2364 (2016).

J. P. Freidberg, Ideal MHD (Cambridge University Press, 2014).

M. Lingam, G. Miloshevich, and P. J. Morrison, Phys. Lett. A 380, 2400 (2016).

M. Lingam, E. Hirvijoki, D. Pfefferlé, L. Comisso, and A. Bhattacharjee, Phys. Plasmas 24, 042120 (2017).

H. Lammer, Origin and Evolution of Planetary Atmospheres: Implications for Habitability, Springer Briefs in Astronomy (Springer, 2013).

M. H. Acuna, J. E. P. Connerney, P. Wasilewski, R. P. Lin, K. A. Anderson, C. W. Carlson, J. McFadden, D. W. Curtis, D. Mitchell, H. Reme, C. Mazelle, J. A. Sauvaud, C. D'Uston, A. Cros, J. L. Medale, S. J. Bauer, P. Cloutier, M. Mayhew, D. Winterhalter, and N. F. Ness, Science 279, 1676 (1998)

J. Zendejas, A. Segura, and A. C. Raga, Icarus 210, 539 (2010).

K. J. Zahnle and D. C. Catling, Astrophys. J. 843, 122 (2017). M. Lingam and A. Loeb, Int. J. Astrobiol. 17, 116 (2018b).

B. E. Wood, H.-R. Müller, G. P. Zank, J. L. Linsky, and S. Redfield, Astrophys. J. Lett. 628, L143 (2005).

S. R. Cranmer and S. H. Saar, Astrophys. J. 741, 54 (2011).

C. P. Johnstone, M. Güdel, I. Brott, and T. Lüftinger, Astron. Astrophys. 577, A28 (2015).

D. Valencia, R. J. O'Connell, and D. Sasselov, Icarus 181, 545 (2006).

L. Zeng, D. D. Sasselov, and S. B. Jacobsen, Astrophys. J. 819, 127 (2016).

V. S. Airapetian, A. Glocer, G. V. Khazanov, R. O. P. Loyd, K. France, J. Sojka, W. C. Danchi, and M. W. Liemohn, Astrophys. J. Lett. 836, L3 (2017a).

K. Garcia-Sage, A. Glocer, J. J. Drake, G. Gronoff, and O. Cohen, Astrophys. J. Lett. 844, L13 (2017).

K. G. Kislyakova, C. P. Johnstone, P. Odert, N. V. Erkaev, H. Lammer, T. Lüftinger, M. Holmström, M. L. Khodachenko, and M. Güdel, Astron. Astrophys. 562, A116 (2014).

O. Cohen, Y. Ma, J. J. Drake, A. Glocer, C. Garraffo, J. M. Bell, and T. I. Gombosi, Astrophys. J. 806, 41 (2015).

K. Seki, R. C. Elphic, M. Hirahara, T. Terasawa, and T. Mukai, Science 291, 1939 (2001).

C. Dong, Z. Huang, M. Lingam, G. Tóth, T. Gombosi, and A. Bhattacharjee, Astrophys. J. Lett. 847, L4 (2017b).

B. K. D. Pearce, A. S. Tupper, R. E. Pudritz, and P. G. Higgs, Astrobiology 18, 343 (2018).

D. S. Spiegel and E. L. Turner, Proc. Natl. Acad. Sci. USA 109, 395 (2012). 
A. J. Rushby, M. W. Claire, H. Osborn, and A. J. Watson, Astrobiology 13, 833 (2013).

M. Lingam and A. Loeb, Int. J. Astrobiol. (2019a), 10.1017/S1473550419000016, arXiv:1804.02271 [physics.pop-ph].

D. A. Russell, Adv. Space Res. 3, 95 (1983).

A. Purvis and A. Hector, Nature 405, 212 (2000).

M. Lingam and A. Loeb, Astrophys. J. Lett. 846, L21 (2017a).

K. J. Locey and J. T. Lennon, Proc. Natl. Acad. Sci. USA 113, 5970 (2016).

M. Lingam and A. Loeb, J. Cosmol. Astropart. Phys. 5, 020 (2018c).

N. V. Erkaev, Y. N. Kulikov, H. Lammer, F. Selsis, D. Langmayr, G. F. Jaritz, and H. K. Biernat, Astron. Astrophys. 472, 329 (2007).

I. Ribas, E. Bolmont, F. Selsis, A. Reiners, J. Leconte, S. N. Raymond, S. G. Engle, E. F. Guinan, J. Morin, M. Turbet, F. Forget, and G. Anglada-Escudé, Astron. Astrophys. 596, A111 (2016).

J. E. Owen, Annu. Rev. Earth Planet. Sci. 47, 67 (2019).

J. E. Owen and M. A. Alvarez, Astrophys. J. 816, 34 (2016).

C. F. McKee and E. C. Ostriker, Annu. Rev. Astron. Astrophys. 45, 565 (2007).

I. Baraffe, G. Chabrier, F. Allard, and P. H. Hauschildt, Astron. Astrophys. 382, 563 (2002).

R. M. Ramirez and L. Kaltenegger, Astrophys. J. Lett. 797, L25 (2014).

R. Luger and R. Barnes, Astrobiology 15, 119 (2015).

E. Bolmont, F. Selsis, J. E. Owen, I. Ribas, S. N. Raymond, J. Leconte, and M. Gillon, Mon. Not. R. Astron. Soc. 464, 3728 (2017).

F. Tian, M. Güdel, C. P. Johnstone, H. Lammer, R. Luger, and P. Odert, Space Sci. Rev. 214, 65 (2018).

F. Tian, Earth Planet. Sci. Lett. 432, 126 (2015b).

H. D. Holland, Geochim. Cosmochim. Acta 66, 3811 (2002).

A. Segura, V. S. Meadows, J. F. Kasting, D. Crisp, and M. Cohen, Astron. Astrophys. 472, 665 (2007).

R. D. Wordsworth and R. T. Pierrehumbert, Astrophys. J. 778, 154 (2013).

R. Wordsworth and R. Pierrehumbert, Astrophys. J. Lett. 785, L20 (2014).

F. Tian, K. France, J. L. Linsky, P. J. D. Mauas, and M. C. Vieytes, Earth Planet. Sci. Lett. 385, 22 (2014).

C. E. Harman, E. W. Schwieterman, J. C. Schottelkotte, and J. F. Kasting, Astrophys. J. 812, 137 (2015).

N. Narita, T. Enomoto, S. Masaoka, and N. Kusakabe, Sci. Rep. 5, 13977 (2015).

P. Gao, R. Hu, T. D. Robinson, C. Li, and Y. L. Yung, Astrophys. J. 806, 249 (2015).

V. S. Meadows, Astrobiology 17, 1022 (2017).

V. S. Meadows, C. T. Reinhard, G. N. Arney, M. N. Parenteau, E. W. Schwieterman, S. D. Domagal-Goldman, A. P. Lincowski, K. R. Stapelfeldt, H. Rauer, S. DasSarma, S. Hegde, N. Narita, R. Deitrick, J. Lustig-Yaeger, T. W. Lyons, N. Siegler, and J. L. Grenfell, Astrobiology 18, 630 (2018b).

V. Bourrier, D. Ehrenreich, P. J. Wheatley, E. Bolmont, M. Gillon, J. de Wit, A. J. Burgasser, E. Jehin, D. Queloz, and A. H. M. J. Triaud, Astron. Astrophys. 599, L3 (2017).

D. P. O'Brien, A. Izidoro, S. A. Jacobson, S. N. Raymond, and D. C. Rubie, Space Sci. Rev. 214, 47 (2018).

S. N. Raymond, J. Scalo, and V. S. Meadows, Astrophys. J. 669, 606 (2007).
G. D. Mulders, F. J. Ciesla, M. Min, and I. Pascucci, Astrophys. J. 807, 9 (2015b).

S. N. Raymond and A. Izidoro, Icarus 297, 134 (2017).

C. T. Unterborn, S. J. Desch, N. R. Hinkel, and A. Lorenzo, Nat. Astron. 2, 297 (2018a).

C. T. Unterborn, N. R. Hinkel, and S. J. Desch, Res. Notes AAS 2, 116 (2018b).

C. Dorn, K. Mosegaard, S. L. Grimm, and Y. Alibert, Astrophys. J. 865, 20 (2018).

L. A. Rogers, Astrophys. J. 801, 41 (2015).

A. Wolfgang and E. Lopez, Astrophys. J. 806, 183 (2015).

J. Chen and D. Kipping, Astrophys. J. 834, 17 (2017).

S. Jin and C. Mordasini, Astrophys. J. 853, 163 (2018).

M. Lozovsky, R. Helled, C. Dorn, and J. Venturini, Astrophys. J. 866, 49 (2018).

Y. Alibert and W. Benz, Astron. Astrophys. 598, L5 (2017).

F. Simpson, Mon. Not. R. Astron. Soc. 468, 2803 (2017).

M. J. Kuchner, Astrophys. J. Lett. 596, L105 (2003).

A. Léger, F. Selsis, C. Sotin, T. Guillot, D. Despois, D. Mawet, M. Ollivier, A. Labèque, C. Valette, F. Brachet, B. Chazelas, and H. Lammer, Icarus 169, 499 (2004).

L. Noack, I. Snellen, and H. Rauer, Space Sci. Rev. 212, 877 (2017).

E. S. Kite and E. B. Ford, Astrophys. J. 864, 75 (2018).

R. M. Ramirez and A. Levi, Mon. Not. R. Astron. Soc. 477, 4627 (2018).

M. M. Hirschmann, Annu. Rev. Earth Planet. Sci. 34, 629 (2006).

J. Korenaga, Terra Nova 20, 419 (2008).

H. Ni, Y.-F. Zheng, Z. Mao, Q. Wang, R.-X. Chen, and L. Zhang, Natl. Sci. Rev. 4, 879 (2017).

Y. Abe, A. Abe-Ouchi, N. H. Sleep, and K. J. Zahnle, Astrobiology 11, 443 (2011).

A. Zsom, S. Seager, J. de Wit, and V. Stamenković, Astrophys. J. 778, 109 (2013).

F. Tian and S. Ida, Nat. Geosci. 8, 177 (2015).

P. S. Zain, G. C. de Elía, M. P. Ronco, and O. M. Guilera, Astron. Astrophys. 609, A76 (2018).

N. F. Hadley and S. R. Szarek, BioScience 31, 747 (1981).

T. Tyrrell, Nature 400, 525 (1999).

A. Paytan and K. McLaughlin, Chem. Rev. 107, 563 (2007).

M. Lingam and A. Loeb, Int. J. Astrobiol. 18, 112 (2019b).

M. Lingam and A. Loeb, Astron. J. 156, 151 (2018d).

M. Lingam and A. Loeb, Astron. J. 157, 25 (2019c).

T. M. McCollom, Annu. Rev. Earth Planet. Sci. 41, 207 (2013).

P. L. Luisi, The Emergence of Life: From Chemical Origins to Synthetic Biology (Cambridge Univ. Press, 2016).

J. D. Sutherland, Angew. Chem. Int. Ed. 55, 104 (2016).

W. Gilbert, Nature 319, 618 (1986).

G. F. Joyce, Nature 418, 214 (2002).

L. E. Orgel, Crit. Rev. Biochem. Mol. Biol. 39, 99 (2004).

M. Neveu, H.-J. Kim, and S. A. Benner, Astrobiology 13, 391 (2013).

P. G. Higgs and N. Lehman, Nat. Rev. Genet. 16, 7 (2015).

G. Wächtershäuser, Chem. Biodivers. 4, 584 (2007).

K. Ruiz-Mirazo, C. Briones, and A. de la Escosura, Chem. Rev. 114, 285 (2014).

J. E. Goldford and D. Segrè, Curr. Opin. Syst. Biol. 4, 117 (2018).

E. E. Stüeken, R. E. Anderson, J. S. Bowman, W. J. Brazelton, J. Colangelo-Lillis, A. D. Goldman, S. M. Som, and J. A. Baross, Geobiology 11, 101 (2013).

N. Kitadai and S. Maruyama, Geosci. Front. 9, 1117 (2018). 
A. S. Romer, Man and the vertebrates (The University of Chicago Press., 1933).

R. Lathe, Icarus 168, 18 (2004).

S. A. Balbus, Proc. R. Soc. London Ser. A 470, 20140263 (2014).

M. Lingam and A. Loeb, Astrobiology 18, 967 (2018e).

S. A. Benner, H.-J. Kim, and M. A. Carrigan, Acc. Chem. Res. 45, 2025 (2012).

A. Y. Mulkidjanian, A. Y. Bychkov, D. V. Dibrova, M. Y. Galperin, and E. V. Koonin, Proc. Natl. Acad. Sci. USA 109, E821 (2012).

W. Martin, J. Baross, D. Kelley, and M. J. Russell, Nat. Rev. Microbiol. 6, 805 (2008).

V. Sojo, B. Herschy, A. Whicher, E. Camprubí, and N. Lane, Astrobiology 16, 181 (2016).

H. J. Cleaves, A. M. Scott, F. C. Hill, J. Leszczynski, N. Sahai, and R. Hazen, Chem. Soc. Rev. 41, 5502 (2012).

R. M. Hazen, Phil. Trans. R. Soc. A 375, 20160353 (2017).

L. Noack, D. Höning, A. Rivoldini, C. Heistracher, N. Zimov, B. Journaux, H. Lammer, T. Van Hoolst, and J. H. Bredehöft, Icarus 277, 215 (2016).

C. Sagan and B. N. Khare, Science 173, 417 (1971).

J. D. Sutherland, Nat. Rev. Chem. 1, 0012 (2017).

T. Gustavsson, R. Improta, and D. Markovitsi, J. Phys. Chem. Lett. 1, 2025 (2010).

J. E. Šponer, R. Szabla, R. W. Góra, A. M. Saitta, F. Pietrucci, F. Saija, E. Di Mauro, R. Saladino, M. Ferus, S. Civiš, and J. Šponer, Phys. Chem. Chem. Phys. 18, 20047 (2016).

D. V. Dibrova, M. Y. Chudetsky, M. Y. Galperin, E. V. Koonin, and A. Y. Mulkidjanian, Orig. Life Evol. Biosph. 42, 459 (2012).

M. W. Powner, B. Gerland, and J. D. Sutherland, Nature 459, 239 (2009).

S. Islam and M. W. Powner, Chem 2, 470 (2017).

D. Ritson and J. D. Sutherland, Nat. Chem. 4, 895 (2012).

D. J. Ritson and J. D. Sutherland, Angew. Chem. Int. Ed. 52, 5845 (2013).

Z. R. Todd, A. C. Fahrenbach, C. J. Magnani, S. Ranjan, A. Björkbom, J. W. Szostak, and D. D. Sasselov, Chem. Commun. 54, 1121 (2018).

B. H. Patel, C. Percivalle, D. J. Ritson, C. D. Duffy, and J. D. Sutherland, Nat. Chem. 7, 301 (2015).

J. Xu, D. J. Ritson, S. Ranjan, Z. R. Todd, D. D. Sasselov, and J. D. Sutherland, Chem. Commun. 54, 5566 (2018).

C. Bonfio, L. Valer, S. Scintilla, S. Shah, D. J. Evans, L. Jin, J. W. Szostak, D. D. Sasselov, J. D. Sutherland, and S. S. Mansy, Nat. Chem. 9, 1229 (2017).

A. C. Rios and Y. Tor, Isr. J. Chem. 53, 469 (2013).

A. A. Beckstead, Y. Zhang, M. S. de Vries, and B. Kohler, Phys. Chem. Chem. Phys. 18, 24228 (2016).

S. Ranjan and D. D. Sasselov, Astrobiology 16, 68 (2016).

S. Rugheimer, A. Segura, L. Kaltenegger, and D. Sasselov, Astrophys. J. 806, 137 (2015a).

S. Ranjan, R. Wordsworth, and D. D. Sasselov, Astrophys. J. 843, 110 (2017).

A. P. Buccino, G. A. Lemarchand, and P. J. D. Mauas, Icarus 192, 582 (2007).

J. Guo, F. Zhang, X. Zhang, and Z. Han, Astrophys. Space Sci. 325, 25 (2010).

P. B. Rimmer, J. Xu, S. J. Thompson, E. Gillen, J. D. Sutherland, and D. Queloz, Sci. Adv. 4, eaar3302 (2018).

M. Oishi and H. Kamaya, Astrophys. J. 833, 293 (2016).
D. Voet, W. B. Gratzer, R. A. Cox, and P. Doty, Biopolymers 1, 193 (1963).

C. Sagan, J. Theor. Biol. 39, 195 (1973).

A. H. Teramura and J. H. Sullivan, Photosynth. Res. 39, 463 (1994).

J. Cadet, E. Sage, and T. Douki, Mutat. Res. 571, 3 (2005).

L. J. Rothschild, J. Eukaryot. Microbiol. 46, 548 (1999).

K. L. Evans and K. J. Gaston, Funct. Ecol. 19, 899 (2005).

H. J. Cleaves and S. L. Miller, Proc. Natl. Acad. Sci. USA 95, 7260 (1998).

G. Arney, S. D. Domagal-Goldman, V. S. Meadows, E. T. Wolf, E. Schwieterman, B. Charnay, M. Claire, E. Hébrard, and M. G. Trainer, Astrobiology 16, 873 (2016).

C. S. Cockell and J. Knowland, Biol. Rev. 74, 311 (1999).

Q. Gao and F. Garcia-Pichel, Nat. Rev. Microbiol. 9, 791 (2011).

P. Gabani and O. V. Singh, Appl. Microbiol. Biotechnol. 97, 993 (2013).

C. Pacelli, R. A. Bryan, S. Onofri, L. Selbmann, I. Shuryak, and E. Dadachova, Environ. Microbiol. 19, 1612 (2017).

K.-W. Jung, S. Lim, and Y.-S. Bahn, J. Microbiol. 55, 499 (2017).

J. T. O'Malley-James and L. Kaltenegger, Mon. Not. R. Astron. Soc. Lett. 469, L26 (2017).

J. T. O'Malley-James and L. Kaltenegger, Mon. Not. R. Astron. Soc. 485, 5598âĂŞ5603 (2019).

M. F. Hohmann-Marriott and R. E. Blankenship, Annu. Rev. Plant Biol. 62, 515 (2011).

N. Y. Kiang, J. Siefert, Govindjee, and R. E. Blankenship, Astrobiology 7, 222 (2007a).

W. Bains, S. Seager, and A. Zsom, Life 4, 716 (2014).

S. Seager, E. L. Turner, J. Schafer, and E. B. Ford, Astrobiology 5, 372 (2005).

L. Kaltenegger, Annu. Rev. Astron. Astrophys. 55, 433 (2017).

N. Y. Kiang, A. Segura, G. Tinetti, Govindjee, R. E. Blankenship, M. Cohen, J. Siefert, D. Crisp, and V. S. Meadows, Astrobiology 7, 252 (2007b).

K. Takizawa, J. Minagawa, M. Tamura, N. Kusakabe, and N. Narita, Sci. Rep. 7, 7561 (2017).

W. B. Sparks, J. Hough, T. A. Germer, F. Chen, S. DasSarma, P. DasSarma, F. T. Robb, N. Manset, L. Kolokolova, N. Reid, F. D. Macchetto, and W. Martin, Proc. Natl. Acad. Sci. USA 106, 7816 (2009).

S. V. Berdyugina, J. R. Kuhn, D. M. Harrington, T. ŠantlTemkiv, and E. J. Messersmith, Int. J. Astrobiol. 15, 45 (2016).

C. E. Doughty and A. Wolf, Astrobiology 10, 869 (2010).

W. W. Fischer, J. Hemp, and J. E. Johnson, Annu. Rev. Earth Planet. Sci. 44, 647 (2016).

J. Gale and A. Wandel, Int. J. Astrobiol. 16, 1 (2017).

R. J. Ritchie, A. W. D. Larkum, and I. Ribas, Int. J. Astrobiol. 17, 147 (2018).

O. R. Lehmer, D. C. Catling, M. N. Parenteau, and T. M. Hoehler, Astrophys. J. 859, 171 (2018).

M. Lingam and A. Loeb, Mon. Not. R. Astron. Soc. 485, 5924 (2019d).

A. H. Knoll, in The Search for Extraterrestrial Life: Recent Developments, IAU Symposium, Vol. 112, edited by M. D. Papagiannis (International Astronomical Union, 1985) pp. 201-211.

M. A. O'Malley and R. Powell, Biol. Philos. 31, 159 (2016).

O. P. Judson, Nat. Ecol. Evol. 1, 0138 (2017).

L. G. Koch and S. L. Britton, J. Physiol. 586, 83 (2008). 
A. P. Gumsley, K. R. Chamberlain, W. Bleeker, U. Söderlund, M. O. de Kock, E. R. Larsson, and A. Bekker, Proc. Natl. Acad. Sci. USA 114, 1811 (2017).

A. H. Knoll and M. A. Nowak, Sci. Adv. 3, e1603076 (2017). J. F. Kasting, Chem. Geol. 362, 13 (2013).

T. W. Lyons, C. T. Reinhard, and N. J. Planavsky, Nature 506, 307 (2014).

D. C. Catling, K. J. Zahnle, and C. P. McKay, Science 293, 839 (2001).

D. C. Catling and J. F. Kasting, Atmospheric Evolution on Inhabited and Lifeless Worlds (Cambridge University Press, 2017).

J. L. Linsky, K. France, and T. Ayres, Astrophys. J. 766, 69 (2013).

M. Livio, Astrophys. J. 511, 429 (1999).

A. O. Benz, Living Rev. Sol. Phys. 14, 2 (2017).

E. R. Priest and T. G. Forbes, Astron. Astrophys. Rev. 10, 313 (2002).

D. Biskamp, Magnetic Reconnection in Plasmas, Cambridge Monographs on Plasma Physics, Vol. 3 (Cambridge University Press, 2000).

K. Shibata and T. Magara, Living Rev. Sol. Phys. 14, 6 (2011).

L. Comisso, M. Lingam, Y.-M. Huang, and A. Bhattacharjee, Phys. Plasmas 23, 100702 (2016).

R. C. Carrington, Mon. Not. R. Astron. Soc. 20, 13 (1859).

E. W. Cliver and W. F. Dietrich, J. Space Weather Space Clim. 3, A31 (2013).

H. Maehara, T. Shibayama, S. Notsu, Y. Notsu, T. Nagao, S. Kusaba, S. Honda, D. Nogami, and K. Shibata, Nature 485, 478 (2012).

T. Shibayama, H. Maehara, S. Notsu, Y. Notsu, T. Nagao, S. Honda, T. T. Ishii, D. Nogami, and K. Shibata, Astrophys. J. Suppl. 209, 5 (2013).

J. R. A. Davenport, Astrophys. J. 829, 23 (2016).

K. Namekata, T. Sakaue, K. Watanabe, A. Asai, H. Maehara, Y. Notsu, S. Notsu, S. Honda, T. T. Ishii, K. Ikuta, D. Nogami, and K. Shibata, Astrophys. J. 851, 91 (2017).

Y. Notsu, H. Maehara, S. Honda, S. L. Hawley, J. R. A. Davenport, K. Namekata, S. Notsu, K. Ikuta, D. Nogami, and K. Shibata, Astrophys. J. 876, 58 (2019).

H. Maehara, T. Shibayama, Y. Notsu, S. Notsu, S. Honda, D. Nogami, and K. Shibata, Earth, Planets, and Space 67, 59 (2015).

M. N. Günther, Z. Zhan, S. Seager, P. B. Rimmer, S. Ranjan, K. G. Stassun, R. J. Oelkers, T. Daylan, E. Newton, E. Gillen, S. Rappaport, G. R. Ricker, D. W. Latham, J. N. Winn, J. M. Jenkins, A. Glidden, M. Fausnaugh, A. M. Levine, J. A. Dittmann, S. N. Quinn, A. Krishnamurthy, and E. B. Ting, Astrophys. J. (2019), arXiv:1901.00443 [astro-ph.EP].

I. G. Hannah, H. S. Hudson, M. Battaglia, S. Christe, J. Kašparová, S. Krucker, M. R. Kundu, and A. Veronig, Space Sci. Rev. 159, 263 (2011).

K. Shibata, H. Isobe, A. Hillier, A. R. Choudhuri, H. Maehara, T. T. Ishii, T. Shibayama, S. Notsu, Y. Notsu, T. Nagao, S. Honda, and D. Nogami, Publ. Astron. Soc. Jpn 65, 49 (2013).

F. Miyake, K. Nagaya, K. Masuda, and T. Nakamura, Nature 486, 240 (2012).

F. Miyake, K. Masuda, and T. Nakamura, Nat. Commun. 4, 1748 (2013).

C. J. Schrijver, J. Beer, U. Baltensperger, E. W. Cliver, M. Güdel, H. S. Hudson, K. G. McCracken, R. A. Osten,
T. Peter, D. R. Soderblom, I. G. Usoskin, and E. W. Wolff, J. Geophys. Res. A 117, A08103 (2012).

M. Lingam and A. Loeb, Astrophys. J. 848, 41 (2017b).

J. R. A. Davenport, D. M. Kipping, D. Sasselov, J. M. Matthews, and C. Cameron, Astrophys. J. Lett. 829, L31 (2016).

K. Vida, Z. Kővári, A. Pál, K. Oláh, and L. Kriskovics, Astrophys. J. 841, 124 (2017).

W. S. Howard, M. A. Tilley, H. Corbett, A. Youngblood, R. O. P. Loyd, J. K. Ratzloff, N. M. Law, O. Fors, D. del Ser, E. L. Shkolnik, C. Ziegler, E. E. Goeke, A. D. Pietraallo, and J. Haislip, Astrophys. J. Lett. 860, L30 (2018).

M. A. MacGregor, A. J. Weinberger, D. J. Wilner, A. F. Kowalski, and S. R. Cranmer, Astrophys. J. Lett. 855, L2 (2018).

M. J. Heath, L. R. Doyle, M. M. Joshi, and R. M. Haberle, Orig. Life Evol. Biosph. 29, 405 (1999).

D. S. Smith, J. Scalo, and J. C. Wheeler, Icarus 171, 229 (2004a).

A. Segura, L. M. Walkowicz, V. Meadows, J. Kasting, and S. Hawley, Astrobiology 10, 751 (2010).

R. Estrela and A. Valio, Astrobiology 18, 1414 (2018).

D. J. Mullan and H. P. Bais, Astron. J. 865, 101 (2018).

C. Scharf, Astrophys. J. 876, 16 (2019).

D. S. Smith, J. Scalo, and J. C. Wheeler, Orig. Life Evol. Biosph. 34, 513 (2004b).

D. F. Webb and T. A. Howard, Living Rev. Sol. Phys. 9, 3 (2012).

E. Kilpua, H. E. J. Koskinen, and T. I. Pulkkinen, Living Rev. Sol. Phys. 14, 5 (2017).

C. M. Ngwira, A. Pulkkinen, M. M. Kuznetsova, and A. Glocer, J. Geophys. Res. A 119, 4456 (2014).

C. Dong, Y. Ma, S. W. Bougher, G. Toth, A. F. Nagy, J. S. Halekas, Y. Dong, S. M. Curry, J. G. Luhmann, D. Brain, J. E. P. Connerney, J. Espley, P. Mahaffy, M. Benna, J. P. McFadden, D. L. Mitchell, G. A. DiBraccio, R. J. Lillis, B. M. Jakosky, and J. M. Grebowsky, Geophys. Res. Lett. 42, 9103 (2015).

B. M. Jakosky, J. M. Grebowsky, J. G. Luhmann, J. Connerney, F. Eparvier, R. Ergun, J. Halekas, D. Larson, P. Mahaffy, J. McFadden, D. F. Mitchell, N. Schneider, R. Zurek, S. Bougher, D. Brain, Y. J. Ma, C. Mazelle, L. Andersson, D. Andrews, D. Baird, D. Baker, J. M. Bell, M. Benna, M. Chaffin, P. Chamberlin, Y.-Y. Chaufray, J. Clarke, G. Collinson, M. Combi, F. Crary, T. Cravens, M. Crismani, S. Curry, D. Curtis, J. Deighan, G. Delory, R. Dewey, G. DiBraccio, C. Dong, Y. Dong, P. Dunn, M. Elrod, S. England, A. Eriksson, J. Espley, S. Evans, X. Fang, M. Fillingim, K. Fortier, C. M. Fowler, J. Fox, H. Gröller, S. Guzewich, T. Hara, Y. Harada, G. Holsclaw, S. K. Jain, R. Jolitz, F. Leblanc, C. O. Lee, Y. Lee, F. Lefevre, R. Lillis, R. Livi, D. Lo, M. Mayyasi, W. McClintock, T. McEnulty, R. Modolo, F. Montmessin, M. Morooka, A. Nagy, K. Olsen, W. Peterson, A. Rahmati, S. Ruhunusiri, C. T. Russell, S. Sakai, J.-A. Sauvaud, K. Seki, M. Steckiewicz, M. Stevens, A. I. F. Stewart, A. Stiepen, S. Stone, V. Tenishev, E. Thiemann, R. Tolson, D. Toublanc, M. Vogt, T. Weber, P. Withers, T. Woods, and R. Yelle, Science 350, 0210 (2015).

C. Kay, M. Opher, and M. Kornbleuth, Astrophys. J. 826, 195 (2016).

M. L. Khodachenko, I. Ribas, H. Lammer, J.-M. Grießmeier, M. Leitner, F. Selsis, C. Eiroa, A. Hanslmeier, H. K. Bier- 
nat, C. J. Farrugia, and H. O. Rucker, Astrobiology 7, 167 (2007).

H. Lammer, H. I. M. Lichtenegger, Y. N. Kulikov, J.-M. Grießmeier, N. Terada, N. V. Erkaev, H. K. Biernat, M. L. Khodachenko, I. Ribas, T. Penz, and F. Selsis, Astrobiology 7, 185 (2007).

P. Odert, M. Leitzinger, A. Hanslmeier, and H. Lammer, Mon. Not. R. Astron. Soc. 472, 876 (2017).

J. D. Alvarado-Gómez, J. J. Drake, O. Cohen, S. P. Moschou, and C. Garraffo, Astrophys. J. 862, 93 (2018).

J. J. Drake, O. Cohen, S. Yashiro, and N. Gopalswamy, Astrophys. J. 764, 170 (2013).

S. R. Cranmer, Astrophys. J. 840, 114 (2017).

D. V. Reames, Space Sci. Rev. 175, 53 (2013).

M. Desai and J. Giacalone, Living Rev. Sol. Phys. 13, 3 (2016).

R. A. Mewaldt, Space Sci. Rev. 124, 303 (2006).

T. Takahashi, Y. Mizuno, and K. Shibata, Astrophys. J. Lett. 833, L8 (2016).

A. Youngblood, K. France, R. O. P. Loyd, A. Brown, J. P. Mason, P. C. Schneider, M. A. Tilley, Z. K. Berta-Thompson, A. Buccino, C. S. Froning, S. L. Hawley, J. Linsky, P. J. D. Mauas, S. Redfield, A. Kowalski, Y. Miguel, E. R. Newton, S. Rugheimer, A. Segura, A. Roberge, and M. Vieytes, Astrophys. J. 843, 31 (2017).

H. S. Hudson, J. Phys. Conf. Ser. 632, 012058 (2015).

I. G. Usoskin, Living Rev. Sol. Phys. 14, 3 (2017).

P. J. Crutzen, Annu. Rev. Earth Planet. Sci. 7, 443 (1979).

M. López-Puertas, B. Funke, S. Gil-López, T. von Clarmann, G. P. Stiller, M. Höpfner, S. Kellmann, H. Fischer, and C. H. Jackman, J. Geophys. Res. A 110, A09S43 (2005).

S. Solomon, Rev. Geophys. 37, 275 (1999).

P. J. Crutzen, I. S. A. Isaksen, and G. C. Reid, Science 189, 457 (1975).

M. A. Tilley, A. Segura, V. S. Meadows, S. Hawley, and J. Davenport, Astrobiology 19, 64 (2019).

S. Rugheimer, L. Kaltenegger, A. Zsom, A. Segura, and D. Sasselov, Astrobiology 13, 251 (2013).

S. Rugheimer, L. Kaltenegger, A. Segura, J. Linsky, and S. Mohanty, Astrophys. J. 809, 57 (2015b).

F. Tabataba-Vakili, J. L. Grenfell, J.-M. Grießmeier, and H. Rauer, Astron. Astrophys. 585, A96 (2016).

E. Böhm-Vitense, Introduction to stellar astrophysics, Vol. 3 (Cambridge University Press, 1992).

D. Atri and A. L. Melott, Astropar. Phys. 53, 186 (2014).

D. Atri, Mon. Not. R. Astron. Soc. Lett. 465, L34 (2017).

S. L. Miller and H. C. Urey, Science 130, 245 (1959).

C. Chyba and C. Sagan, Nature 355, 125 (1992).

D. Deamer and A. L. Weber, Cold Spring Harb. Perspect. Biol. 2, a004929 (2010).

V. S. Airapetian, A. Glocer, G. Gronoff, E. Hébrard, and W. Danchi, Nat. Geosci. 9, 452 (2016).

M. L. Wong, B. D. Charnay, P. Gao, Y. L. Yung, and M. J. Russell, Astrobiology 17, 975 (2017).

S. Ranjan, Z. R. Todd, P. B. Rimmer, D. D. Sasselov, and A. R. Babbin, Geochem. Geophys. Geosys. 20, 2021 (2019).
K. Kobayashi, T. Kaneko, T. Saito, and T. Oshima, Orig. Life Evol. Biosph. 28, 155 (1998).

S. Miyakawa, H. Yamanashi, K. Kobayashi, H. J. Cleaves, and S. L. Miller, Proc. Natl. Acad. Sci. USA 99, 14628 (2002).

M. Lingam, C. Dong, X. Fang, B. M. Jakosky, and A. Loeb, Astrophys. J. 853, 10 (2018).

F. Fraschetti, J. J. Drake, J. D. Alvarado-Gómez, S. P. Moschou, C. Garraffo, and O. Cohen, Astrophys. J. 874, 21 (2019).

J. M. Nava-Sedeño, A. Ortiz-Cervantes, A. Segura, and S. D. Domagal-Goldman, Astrobiology 16, 744 (2016).

C. Sagan and G. Mullen, Science 177, 52 (1972).

G. Feulner, Rev. Geophys. 50, RG2006 (2012).

C. Voigt, M. E. Marushchak, R. E. Lamprecht, M. JackowiczKorczyński, A. Lindgren, M. Mastepanov, L. Granlund, T. R. Christensen, T. Tahvanainen, P. J. Martikainen, and C. Biasi, Proc. Natl. Acad. Sci. USA 114, 6238 (2017).

D. E. Canfield, A. N. Glazer, and P. G. Falkowski, Science 330, 192 (2010).

V. S. Airapetian, C. H. Jackman, M. Mlynczak, W. Danchi, and L. Hunt, Sci. Rep. 7, 14141 (2017b).

J. A. Robles, C. H. Lineweaver, D. Grether, C. Flynn, C. A. Egan, M. B. Pracy, J. Holmberg, and E. Gardner, Astrophys. J. 684, 691 (2008).

A. Loeb, R. A. Batista, and D. Sloan, J. Cosmol. Astropart. Phys. 8, 040 (2016).

R. Luger, R. Barnes, E. Lopez, J. Fortney, B. Jackson, and V. Meadows, Astrobiology 15, 57 (2015).

H. Chen, J. C. Forbes, and A. Loeb, Astrophys. J. Lett. 855, L1 (2018).

D. Tamayo, H. Rein, C. Petrovich, and N. Murray, Astrophys. J. Lett. 840, L19 (2017).

C. W. Ormel, B. Liu, and D. Schoonenberg, Astron. Astrophys. 604, A1 (2017).

J. H. Steffen and G. Li, Astrophys. J. 816, 97 (2016).

M. Lingam and A. Loeb, Proc. Natl. Acad. Sci. USA 114, $6689(2017 \mathrm{c})$.

S. Krijt, T. J. Bowling, R. J. Lyons, and F. J. Ciesla, Astrophys. J. Lett. 839, L21 (2017).

F. Rodler and M. López-Morales, Astrophys. J. 781, 54 (2014).

I. Snellen, R. de Kok, J. L. Birkby, B. Brandl, M. Brogi, C. Keller, M. Kenworthy, H. Schwarz, and R. Stuik, Astron. Astrophys. 576, A59 (2015).

J. K. Barstow and P. G. J. Irwin, Mon. Not. R. Astron. Soc. Lett. 461, L92 (2016).

C. V. Morley, L. Kreidberg, Z. Rustamkulov, T. Robinson, and J. J. Fortney, Astrophys. J. 850, 121 (2017).

N. Y. Kiang, S. Domagal-Goldman, M. N. Parenteau, D. C. Catling, Y. Fujii, V. S. Meadows, E. W. Schwieterman, and S. I. Walker, Astrobiology 18, 619 (2018).

N. Madhusudhan, Annu. Rev. Astron. Astrophys. (2019), arXiv:1904.03190 [astro-ph.EP]. 